\title{
A Comparison of Coaxial and Conventional Rotor Performance
}

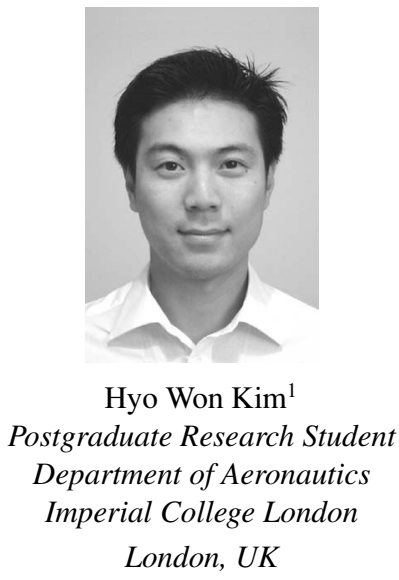

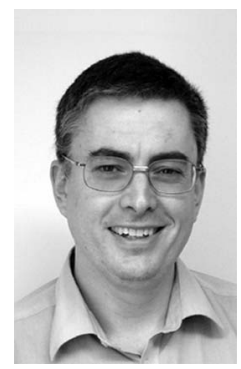

Richard E. Brown* Mechan Chair of Engineering Department of Aerospace Engineering University of Glasgow

Glasgow, UK

\begin{abstract}
The performance of a coaxial rotor in hover, in steady forward flight, and in level, coordinated turns is contrasted with that of an equivalent, conventional rotor with the same overall solidity, number of blades, and blade aerodynamic properties. Brown's vorticity transport model is used to calculate the profile, induced, and parasite contributions to the overall power consumed by the two systems, and the highly resolved representation of the rotor wake that is produced by the model is used to relate the observed differences in the performance of the two systems to the structures of their respective wakes. In all flight conditions, all else being equal, the coaxial system requires less induced power than the conventional system. In hover, the conventional rotor consumes increasingly more induced power than the coaxial rotor as thrust is increased. In forward flight, the relative advantage of the coaxial configuration is particularly evident at pretransitional advance ratios. In turning flight, the benefits of the coaxial rotor are seen at all load factors. The beneficial properties of the coaxial rotor in forward flight and maneuver, as far as induced power is concerned, are a subtle effect of rotor-wake interaction and result principally from differences between the two types of rotor in the character and strength of the localized interaction between the developing supervortices and the highly loaded blade-tips at the lateral extremities of the rotor. In hover, the increased axial convection rate of the tip vortices appears to result in a favorable redistribution of the loading slightly inboard of the tip of the upper rotor of the coaxial system.
\end{abstract}

\section{Nomenclature}

$C_{D} \quad$ sectional profile drag coefficient

$C_{L} \quad$ sectional lift coefficient

$C_{P}$ rotor power coefficient

$C_{T}$ rotor thrust coefficient

$N$ number of blades per rotor

$n$ load factor

$R$ rotor radius

$r \quad$ blade spanwise coordinate

$v_{i} \quad$ induced velocity

$z \quad$ rotor axial coordinate

$\alpha \quad$ section angle of attack

$\gamma$ rotor bank angle/lateral shaft inclination

$\theta_{0} \quad$ collective pitch

$\theta_{1 s} \quad$ longitudinal cyclic pitch

$\theta_{1 c} \quad$ lateral cyclic pitch

\footnotetext{
*Corresponding author; email: r.brown@aero.gla.ac.uk.

Based on a paper presented at the American Helicopter Society 62nd Annual Forum, Phoenix, AZ, May 9-11, 2006. Manuscript received June 2007; accepted July 2009.

${ }^{1}$ Currently a Postdoctoral Research Assistant, Department of Aerospace Engineering, University of Glasgow, Glasgow, UK.
}

\author{
$\mu$ rotor advance ratio \\ $\sigma$ rotor solidity \\ $\psi \quad$ wake age/blade azimuth \\ $\Omega$ rotor rotational speed

$\begin{array}{ll}\text { Subscripts } \\ i & \text { induced component } \\ l & \text { lower rotor of coaxial system } \\ p & \text { profile component } \\ u & \text { upper rotor of coaxial system }\end{array}$

Introduction

Recent developments in the rotorcraft world, led by Sikorsky Aircraft Corporation's announcement of their X2 demonstrator and the development of several UAV prototypes, indicate a resurgence of interest in the coaxial rotor configuration as a technological solution to operational requirements for increased helicopter forward speed, maneuverability, and load-carrying ability.

Note: Throughout this paper, the lower rotor of the coaxial system should be taken to rotate anticlockwise and the upper rotor to rotate clockwise, when viewed from above. In single rotor simulations, the rotor should be taken to rotate anticlockwise when viewed from above. 
The coaxial concept is not new, of course. Although Russia has historically been the world's largest developer and user of coaxial rotor helicopters, and an extensive body of research has been produced in that country, the United States, United Kingdom, Germany, and Japan have also pursued research into the coaxial rotor configuration (Ref. 1). Some highly innovative designs, such as Sikorsky's S-69 Advancing Blade Concept (also known as the XH-59A), and Kamov's Ka-50 attack helicopter, have attempted to exploit the coaxial configuration to obtain improved performance in parts of the flight envelope.

In several cases, however, the performance of practical coaxial configurations fell short of expectations, and this led to a temporary hiatus in the development of the concept. In many such cases, the shortcomings in the practical implementation of the coaxial concept could be traced back to deficiencies in modeling or understanding the specific details of the interaction between the rotors and the effect of the wake on the behavior of the system-especially under unsteady flight conditions. In recent years, though, computational tools have developed to the extent where the highly interactive and nonlinear wake flows generated by the two rotors of the coaxial configuration can be modeled with a much greater degree of confidence than has been possible in the past.

The aim of this paper is to quantify, through numerical simulation, the differences in performance between coaxial and conventional rotor systems in steady flight and during maneuvers, and, in particular, to examine the effect of the differences in the structure and development of their wakes on the performance of the two types of rotor system. The aerodynamic environment of any of the blades of a conventional rotor is strongly influenced by close interactions not only with its own wake but also with the wakes that are generated by the other blades of the rotor. The aerodynamic environment of the rotor blades in a coaxial rotor configuration is further complicated by interactions with the wakes that are generated by the blades on the opposing rotor of the system. Given the relatively long-range nature of these interactions, the numerical modeling of coaxial systems has always posed a significant challenge. This is because adequate resolution of the loading on the blades, hence rotor performance, requires the strength and geometry of the wake of the rotor to be correctly captured and retained for the significant amount of time during which it has an influence on the loading on the system. Adequate resolution of the interrotor interactions that characterize the coaxial rotor system still poses a significant challenge for most current numerical methods because of the prohibitively large computational resources that are required to prevent premature dissipation of the wake.

\section{Computational Model}

Calculations using the vorticity transport model (VTM), developed by Brown (Ref. 2) and extended by Brown and Line (Ref. 3), are used in this paper to expose some of the subtle differences between the wake structures generated by conventional and coaxial rotor systems that lie at the root of the differences in their performance. The VTM has shown considerable success in both capturing and preserving the complex vortex structures contained within the wakes of conventional helicopter rotors (Refs. 2 and 3), and in Ref. 4 the ability of the method also to capture convincingly the aerodynamics of coaxial rotor systems, both in hover and in forward flight, was demonstrated.

The VTM is based on a time-dependent computational solution of the vorticity-velocity form of the Navier-Stokes equations on a Cartesian grid that surrounds the rotorcraft. The problem of preserving the vortical structures in the flow from the effects of numerical dissipation is addressed very effectively by the convection algorithm that is used in the VTM, resulting in a wake structure that remains intact for very large dis- tances downstream of the rotor system. Hence long-range aerodynamic interactions that are produced by wake effects generally tend to be well represented. The VTM uses an adaptive grid system to follow the evolution of the wake. This is done by generating computational cells where vorticity is present and destroying the cells once the vorticity moves elsewhere. The computational domain is thus effectively boundary-free, and significant memory savings are achieved. Computational efficiency is further enhanced by using a series of nested computational grids to capture the wake. The cells within the outer grids are arranged to be coarser than those closer to the rotor. This helps to reduce the overall cell count during a computation while still maintaining a highly resolved flow field near the rotor.

The rotor blades are assumed to be rigid, but the coupled flap-lagfeather dynamics are fully represented through numerical reconstruction of the nonlinear Lagrangian of the system to obtain the equations of motion of the blades. The inertial contributions arising from pitch and roll rates, in the case of maneuvering flight, are accounted for by applying the Lagrangian formulation in the inertial frame of reference. In the version of the VTM used to generate the results presented in this paper, the blade aerodynamics is modeled using an extension of the Weissinger-L version of lifting line theory. Local blade stall is modeled using a variation on Kirchoff's trailing edge separation model, where the length of the stall cell is given as a prescribed function of local angle of attack based on known airfoil characteristics. Since this aerodynamic model is still essentially inviscid, the profile drag of the blade is calculated as a separate function of local angle of attack and then added to the local aerodynamic force that is calculated from the lifting line model.

Throughout the simulations presented in this paper, the computational domain is discretized such that one rotor radius is resolved over 40 grid cells. The computational time step used to evolve the simulations is chosen to be equivalent to $3 \mathrm{deg}$ of the rotor azimuth.

\section{Rotor Model}

In Ref. 4, it was argued that the fairest fundamental comparison between the performance of conventional and coaxial systems would result if the differences in the geometry of the two systems was confined to those characteristics that fundamentally distinguish the two rotor configurations, in other words the vertical separation between the blades and their relative sense of rotation. Any differences in the performance of the two types of rotor should then arise solely as a result of the differences in the detailed interaction between the blades and their wakes that arise within the two types of system. For this reason, in the present work the aerodynamics of the coaxial rotor are compared to those of a conventional, single rotor that has the same blade geometry and overall number of blades. As in Ref. 4, this paper focuses on the characteristics of the coaxial system referred to as "rotor 1" in Harrington's widely regarded experimental comparison of the performance of conventional and coaxial rotors (Ref. 5). This system consisted of two, contrarotating, two-bladed teetering rotors, separated by $0.19 R$ along a shared rotational axis, which were operated at a tip Reynolds number of about $1 \times 10^{6}$. The blades of Harrington's rotors were untwisted, had linear taper, and the blade sections were based on the symmetric NACA 4-digit profile. For modeling purposes, the same spanwise variation in sectional lift and drag coefficients along the span of the blades was assumed to hold for both the conventional and coaxial rotor systems. In all cases the profile drag of the blades was assumed to obey

$$
C_{D}(\alpha)=\max \left[0.0124,0.49\left\{1-\cos 2\left(\alpha+1.75^{\circ}\right)\right\}\right]
$$


where $\alpha$ is the local angle of attack of the blade section. This drag model was extracted from the correlations of VTM predictions against Harrington's experimental data presented in Ref. 4 and is capable of representing the drag rise that is associated with the onset of separation and eventual blade stall. This drag model is used exclusively throughout the simulations presented in this paper to avoid any variability in the profile power from obscuring an argument that is essentially in terms of induced power.

\section{Hover}

The power required by a rotorcraft for a given lifting capacity is determined, in most cases, by the hover performance of its rotor system. For a coaxial rotor, trim of the yawing moment is achieved by matching the torque of the upper and lower rotors via differential collective pitch input so that the net torque about the shared rotor axis is zero. It has long been a point of contention whether or not this arrangement is more efficient than the more conventional main rotor-tail rotor configuration, where typically the tail rotor consumes an additional $5 \%-10 \%$ of the main rotor power to maintain overall yaw moment equilibrium in steady hover (Ref. 6).

In Fig. 1, VTM predictions of the performance of Harrington's twobladed coaxial rotor are compared against predictions of the performance of a four-bladed, conventional (i.e., planar, corotating) rotor configuration. To ensure the strong geometric equivalence that was argued in Ref. 4 to be necessary for direct comparison of the performance of coaxial and conventional systems, the conventional rotor has blades that have the same geometry and aerodynamic properties as the blades of the coaxial rotor. The geometric properties of the two types of rotors are contrasted in Table 1. As a check on the validity of this approach, Fig. 2 shows the similarity in the collective pitch required by the two rotors to trim to a given thrust coefficient that would be expected between rotors that have the same solidity and hence very similar lifting performance. Indeed, a comparison of the performance of the two types of rotor when computed using the same profile drag model, as shown in Fig. 1, reveals the coaxial rotor to consume very similar, albeit consistently less, power than the conventional rotor for the same thrust when this strong geometric and aerodynamic equivalence between the two systems is enforced.

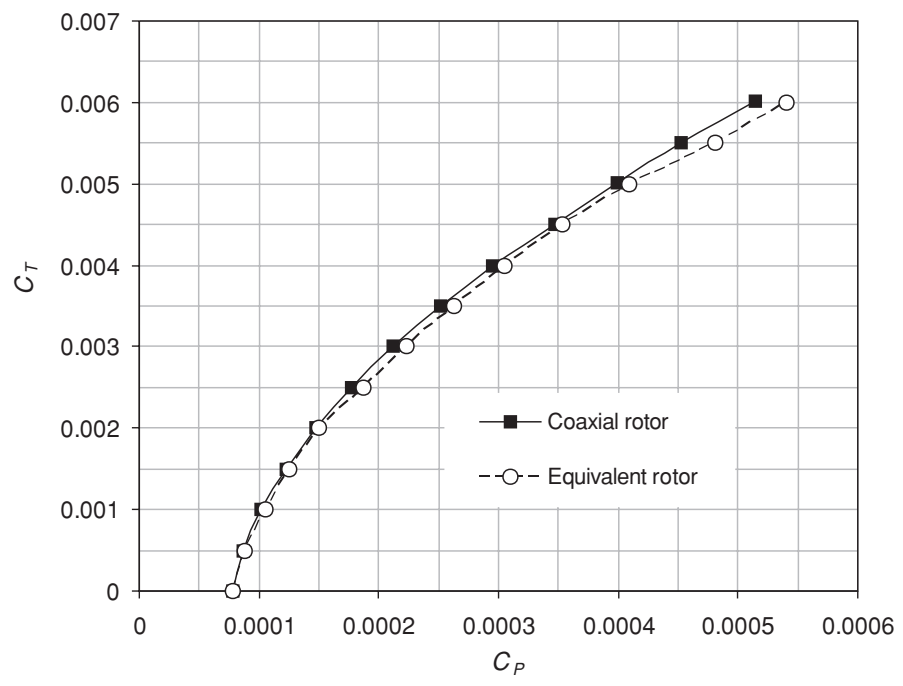

Fig. 1. Power vs. thrust: comparison between rotors with identical solidity and blade properties.
Table 1. Summary of rotor properties

\begin{tabular}{lcc}
\hline \hline & Coaxial Rotor & Equivalent Rotor \\
\hline Rotor radius & $R$ & $R$ \\
Number of rotors & 2 & 1 \\
Blades per rotor & 2 & 4 \\
Rotor separation & $0.190 R$ & $\mathrm{n} / \mathrm{a}$ \\
Root cutout & $0.133 R$ & $0.133 R$ \\
Overall solidity & 0.054 & 0.054 \\
Twist & None & None \\
Flap hinge offset & 0 (teetering) & $0.023 R$ \\
Airfoil sections & NACA-00xx series & NACA-00xx series \\
\hline \hline
\end{tabular}

\section{Wake geometry in hover}

This difference in performance must manifest itself in the geometry of the wakes of the two types of rotor. In Fig. 3, VTM-generated contour maps of vorticity magnitude on a vertical slice through the center of the wake show the global differences between the geometry of the wake of Harrington's coaxial rotor and that of the equivalent four-bladed conventional rotor. Near to the rotors, the images show the orderly downstream procession of the tip vortices and their associated inner wake sheets, the obvious difference between the two systems being the double-tube structure formed by the tip vortices of the coaxial rotor. In both cases, the orderly helicoidal structure of the wake is disrupted, roughly a rotor radius below the rotor plane, as the individual tip vortices coalesce into larger vortical structures. In the case of the coaxial rotor, the tip vortices from both the upper and the lower rotors interact during this process to form a single sequence of coalesced vortical structures. As originally observed by Landgrebe (Ref. 7) in his study of the geometry of the wakes of hovering rotors, the formation of these large structures effectively marks the end of the contraction of the wake and, in fact, the beginning of an expansion in the diameter of the wake as these structures continue to convect downstream of the rotor. Eventually, these structures are themselves torn apart, through their own mutual interaction, to form the extensive field of highly disordered, low-level vorticity in the far-wake that is seen in both images.

Figure 4 compares VTM calculations of the contraction and axial convection rate of the tip vortices generated by Harrington's coaxial rotor system in hover with that of the equivalent, conventional rotor and thus

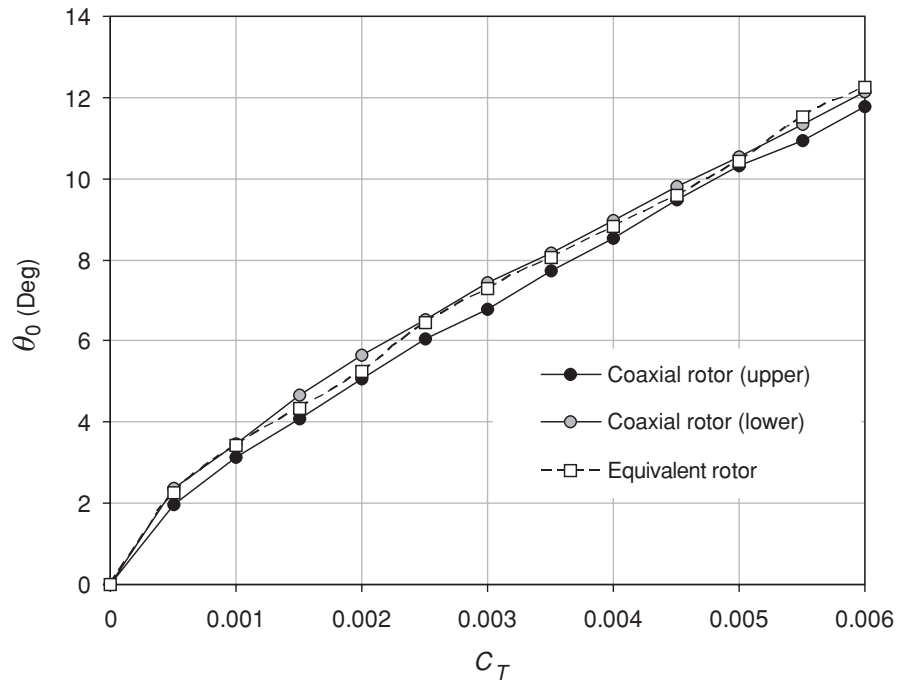

Fig. 2. Collective pitch required to trim to given thrust coefficient. 


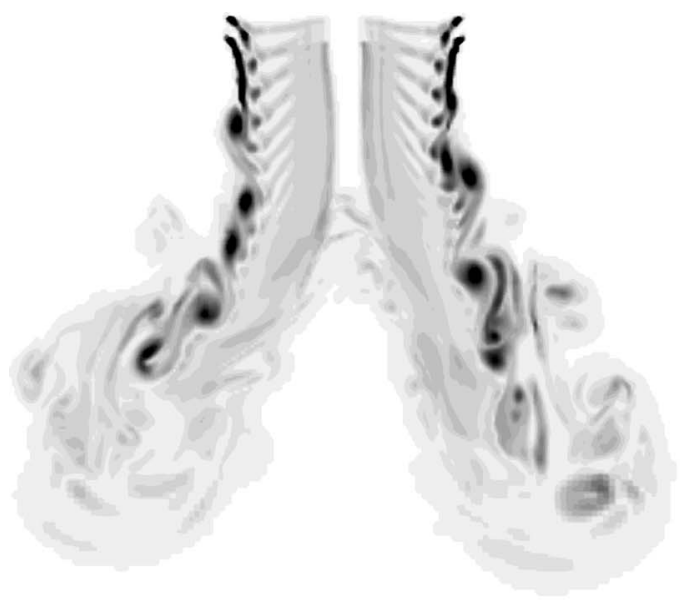

(a) Coaxial rotor

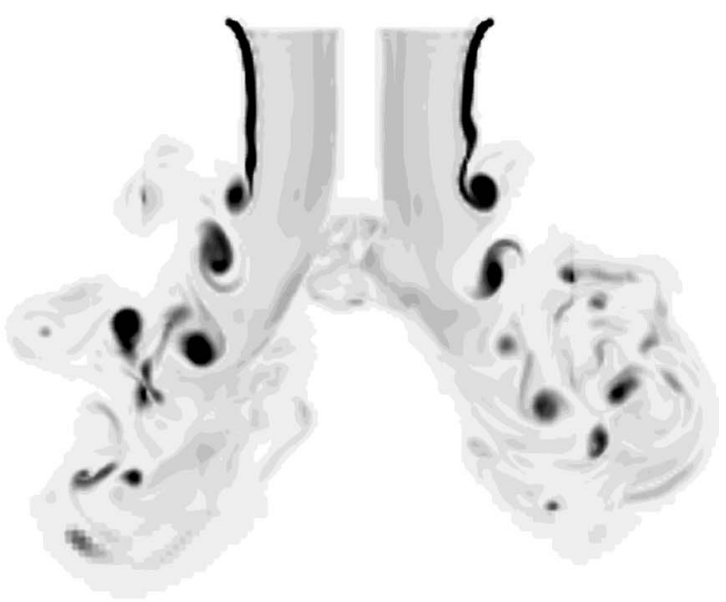

(b) Equivalent conventional rotor

Fig. 3. Wake structure in hover $\left(C_{T}=\mathbf{0 . 0 0 2 5}\right)$.

provides a somewhat more quantitative comparison of the differences in wake structure between the two rotor systems. $\psi$ is the wake age in terms of relative blade azimuth since generation of the tip vortex. The finite resolution of the flow domain yields an estimated error in the calculated positions of the tip vortices of approximately $1 / 40$ of the rotor radius. Langrebe's empirical correlations of wake geometry (Ref. 7) for isolated rotors operating at the same thrust coefficient as the various individual rotors of the conventional and coaxial systems are also plotted to allow the differences between the wakes that are generated by the two systems to be assessed more clearly.

The agreement between Landgrebe's correlation and the VTMpredicted tip-vortex trajectory for the equivalent rotor is extremely close,

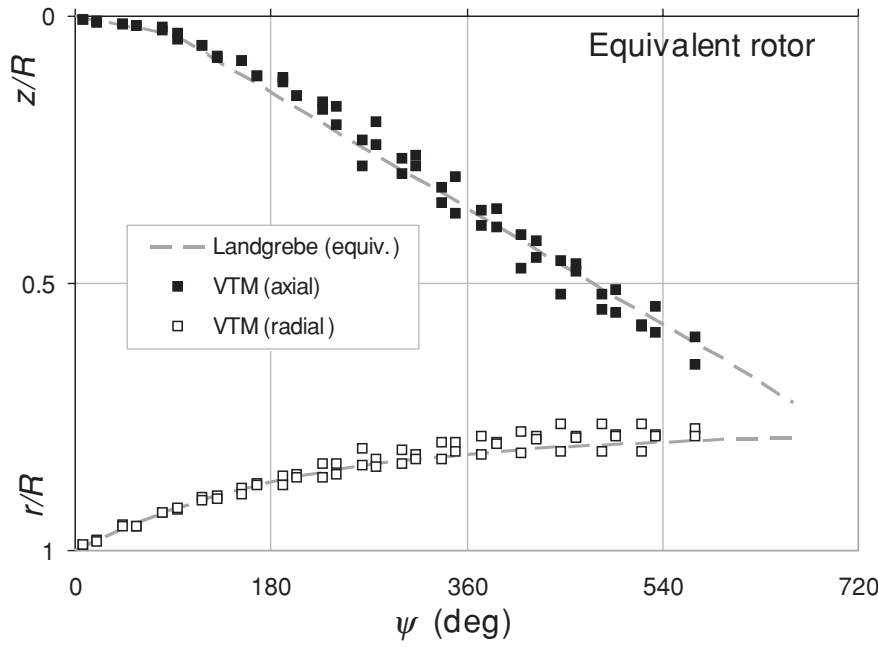

Spatial positions of the tip vortex

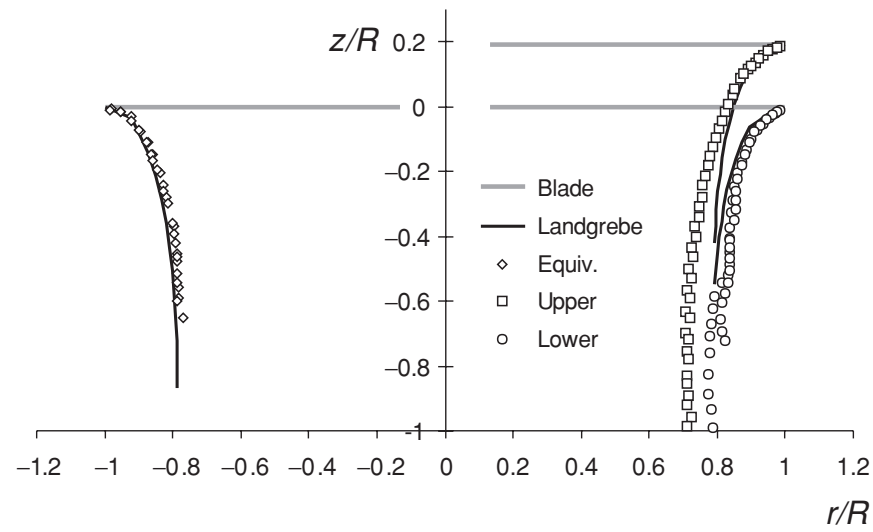

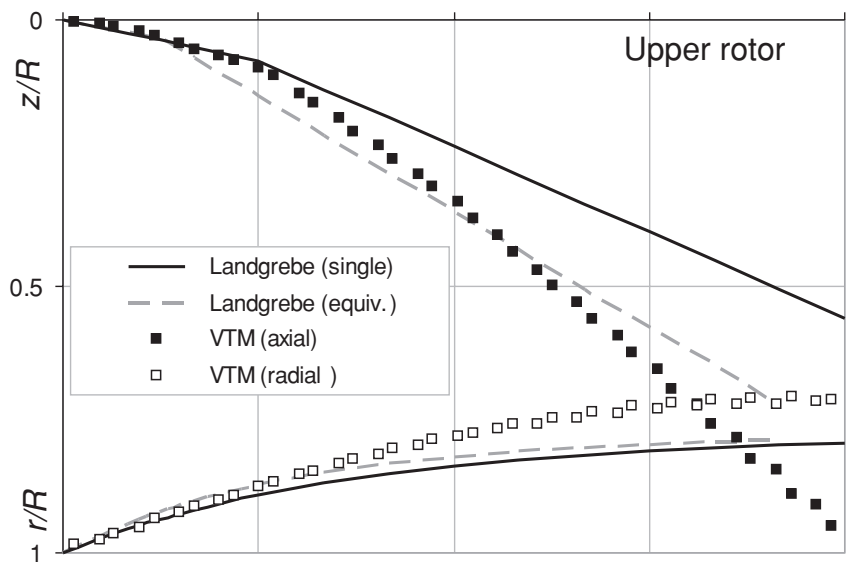

720

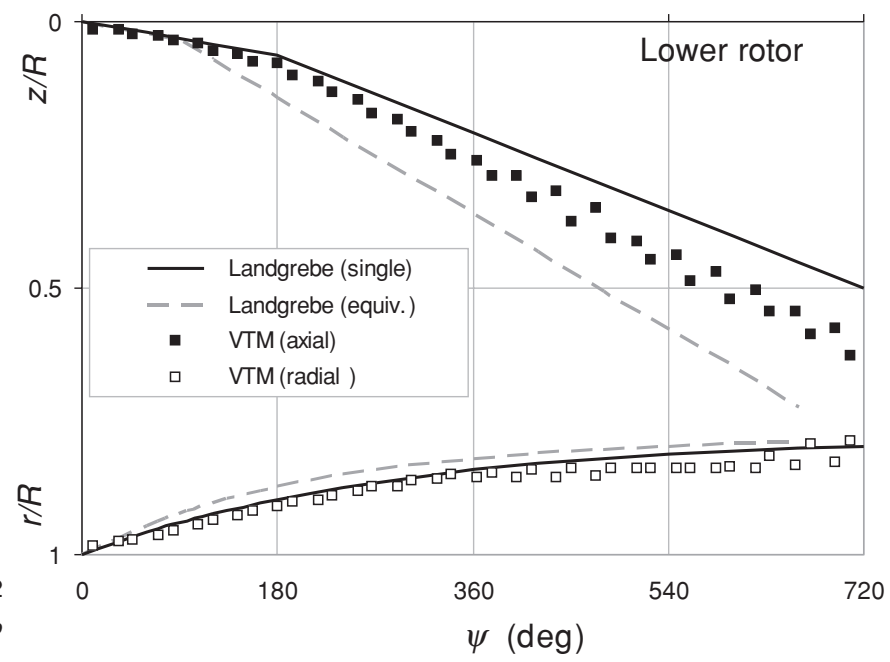

Fig. 4. Correlation of relative tip vortex trajectories against Landgrebe's empirical model (Ref. 7$)$ for the rotors in isolation $\left(C_{T}=0.0048\right)$. 
providing good faith in the quality of the numerical simulations. For the coaxial system, it is immediately obvious that the axial convection rate of the tip vortices that are generated by the upper rotor is greater, and, conversely, for the lower rotor, is smaller, than the axial convection rate of the tip vortices of the equivalent conventional rotor. This observation is consistent with the expected effect of the interaction between the wake structures that are generated by the two rotors of the coaxial system, whereby the convection rate of the vortices from the upper rotor is enhanced by their passage through the region of downwash that is generated by the wake of the lower rotor, and, vice versa, the convection rate of the vortices from the lower rotor is reduced by virtue of their passage through the region of retarded flow lying just outside the wake tube of the upper rotor. Note though that the axial descent rate of the tip vortices of both rotors of the coaxial system is increased compared to the rate at which the vortices would convect if the rotors were to be operated in isolation, as might be expected as a result of the increased overall thrust, and hence the rate of transfer of momentum into the wake, of the combined rotor system.

The rate of radial contraction of the tip vortices that are generated by the upper rotor of the coaxial system is markedly increased compared to that of both the same rotor if operated in isolation and the equivalent conventional rotor. At first glance, the rate of contraction of the tip vortices of the lower rotor, especially when measured in terms of wake age, appears to be largely unaffected by the incorporation of the rotor into the coaxial system. However, bearing in mind that the rate of axial convection of the tip vortices from the lower rotor is higher than when operated in isolation, the contraction of the wake tube that is generated by this rotor is seen to be actually slightly less when incorporated into the coaxial system than when operated in isolation. In this context, the relative geometries of the resultant wake tubes of the coaxial and conventional systems are more clearly visualized in the diagram at bottom left in Fig. 4. Again, these changes in geometry are consistent with the expected form of the mutual interaction between the vortex systems that are generated by the two rotors of the coaxial system.

It should be noted that the VTM provides a fully unsteady computation even when only the trimmed state of the rotor is of interest. Figure 5 shows the unsteadiness of the aerodynamic environment that is experienced by the blades of the rotors when in hover. The individual plots show the radial distribution of inflow that is experienced by a single blade, during a single rotor revolution, as a polar function of blade azimuth. A sharp peak in inflow on the lower rotor of the coaxial system, induced by the nearby passage of the tip vortices from the upper rotor, is clearly visible at a radius of approximately $0.75 R$. The very obvious four-per-revolution character of this interrotor blade-vortex interaction is a consequence of the $2 \mathrm{~N}$-per-revolution geometric periodicity of the system. The upper rotor shows a far more benign variation of inflow along the blade span because this interaction is absent. A weaker, secondary, blade overpressure-type interaction results from the direct influence of the bound vorticity of the blades of the upper rotor on those of the lower, and vice versa, and is visible on both rotors as a ridge of slightly modified inflow over the entire span of the blade at $0,90,180$, and $270 \mathrm{deg}$ azimuth (i.e., when the reference blade passes by one of the blades on the adjacent rotor). In comparison, the bottom plot, for a blade on the isolated, four-bladed equivalent rotor, shows none of these effects to be present, and the resultant inflow distribution to be relatively steady except near the tips of the blades, where the inflow is strongly influenced by the proximity of the tip vortices that are trailed from the preceding blades of the same rotor. The fluctuation in the inflow is associated with a small variability in the trajectory of these vortices that seems to be induced by the unsteadiness in the wake further downstream as the individual tip vortices coalesce to form the larger, less coherent structures described earlier. A similar, but much weaker, fluctuation in the inflow is predicted
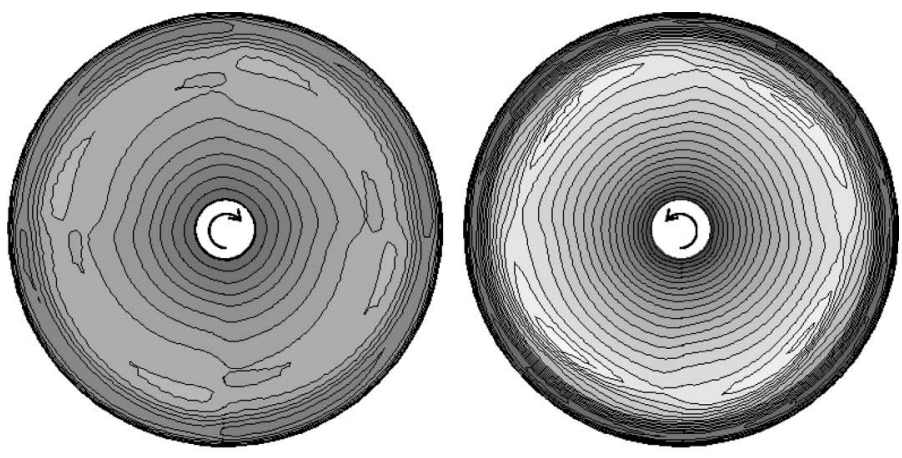

(a) Coaxial rotor (left: upper rotor, right: lower rotor)

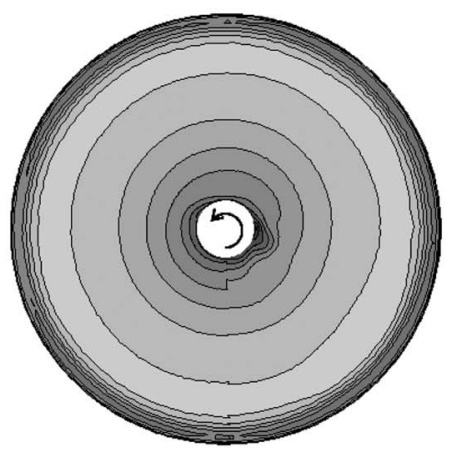

(b) Equivalent conventional rotor

$v_{i} / \Omega R$

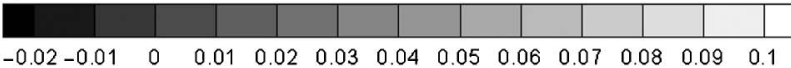

Fig. 5. Spanwise inflow distribution experienced by a single blade during one revolution when in hover (Harrington's rotor, $C_{T}=$ 0.0048).

near the root of the blade where the effects of a similar unsteadiness in the location of the root vortices is most strongly felt.

\section{Hover performance}

Figures 6-8 compare the computed distribution of blade loading (sectional force normal to the blade chord normalized by rotor tip speed), inflow, profile drag, and sectional power loading along the blades of the upper and lower rotors of his coaxial system, one of the rotors of Harrington's system when operated in isolation, and the equivalent four-bladed conventional system, to show in detail how aerodynamic interaction between the wake and the blades subtly modifies the performance of each of the rotor systems. The error bars in the figures represent the variability in the data over a single rotor revolution even when the rotor is ostensibly in a trimmed flight condition.

Calculations are presented for a representative overall thrust coefficient of 0.005 . At this operating condition, the lower rotor is required to generate a thrust coefficient of 0.0022 and the upper rotor a thrust coefficient of 0.0028 to satisfy a trim condition of zero overall torque produced by the rotor system.

Figure 6 compares the upper and lower rotors of the coaxial configuration and exposes the effect of aerodynamic interactions between the two rotors on the performance of the system. Figure 6(b) shows strong distortion of the radial inflow variation along the lower rotor of the coaxial system compared to that along the upper rotor where the distribution of inflow is qualitatively (and quantitatively) not very different from that of one of the rotors of the coaxial configuration tested in isolation at 


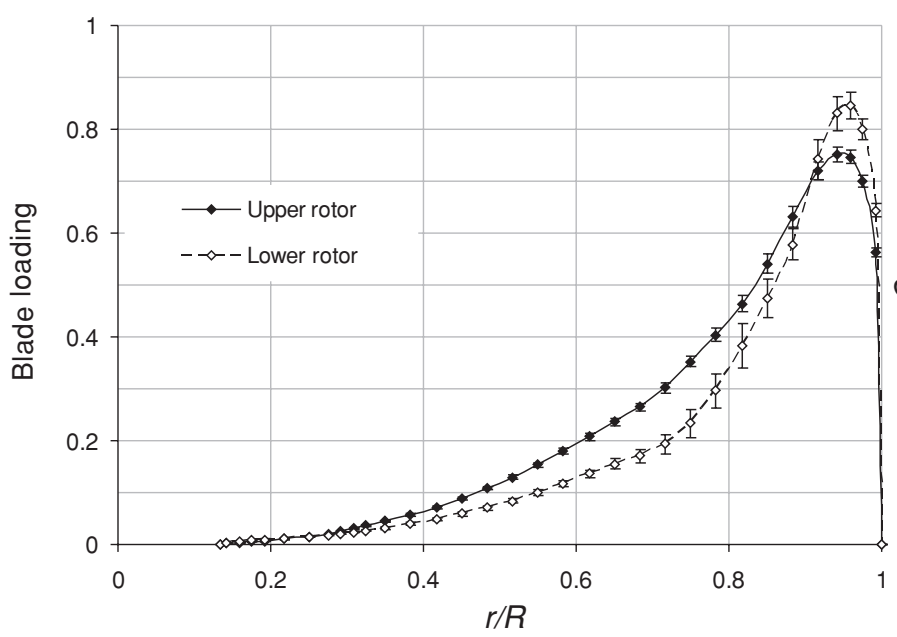

(a) Blade loading

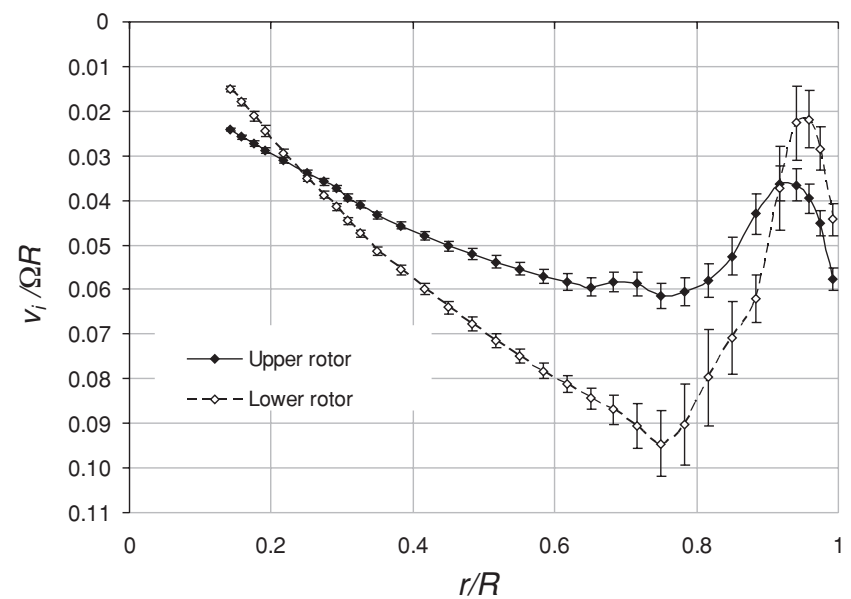

(b) Inflow

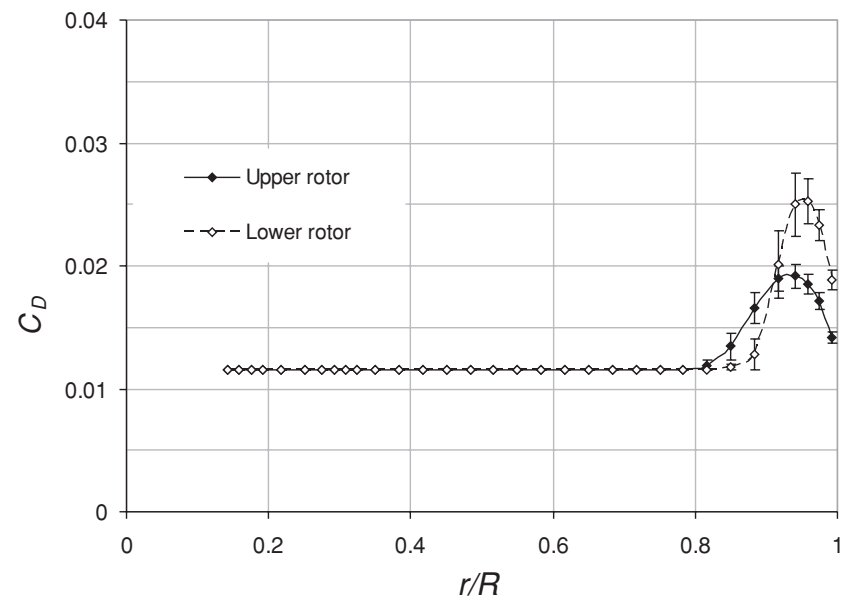

(c) Profile drag

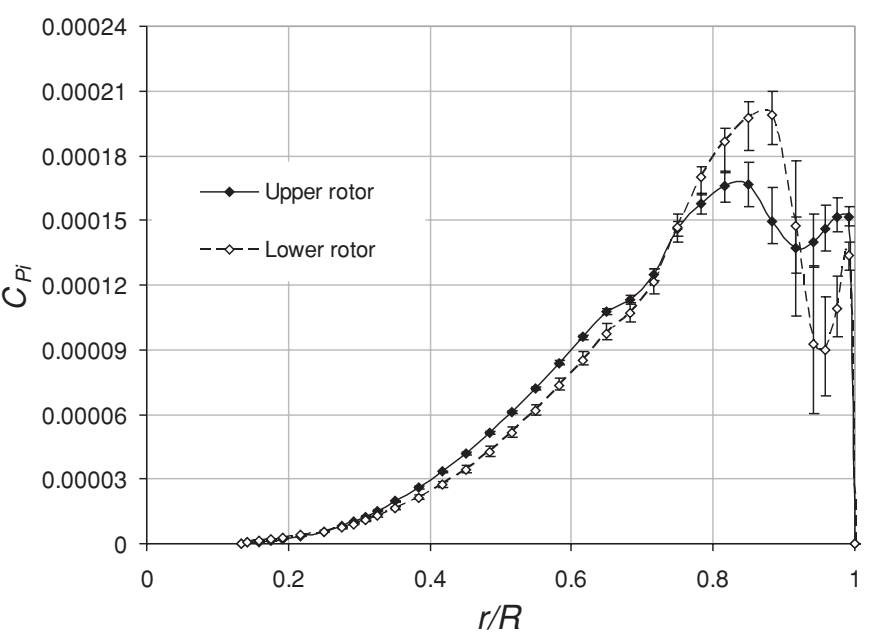

(d) Induced power

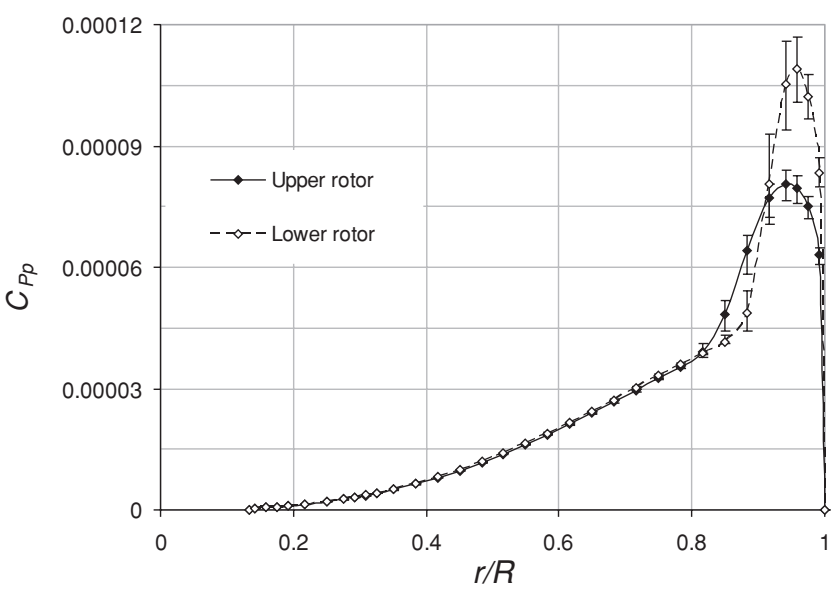

(e) Profile power

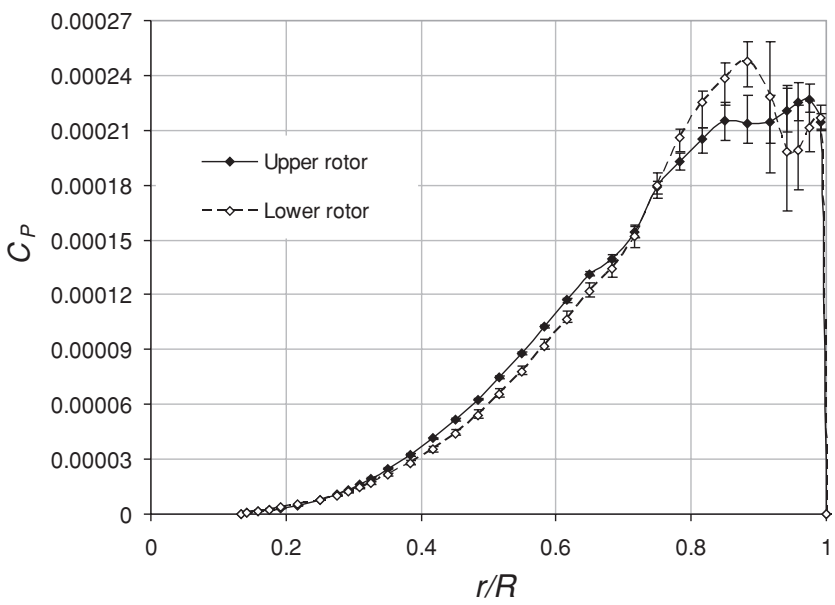

(f) Total power

Fig. 6. Radial distribution of various parameters: upper and lower rotors of coaxial system compared $\left(\right.$ overall $\left.C_{T}=0.0050\right)$.

very similar thrust coefficient as shown in Fig. 7(b). The difference in loading near the tip of the isolated single rotor, compared to the upper rotor of the coaxial system, is entirely consistent with the difference in the tip vortex trajectories of the two systems, as shown earlier, that yields an increased separation between vortex and blade - and hence a reduced effect on the loading on the upper rotor of the coaxial system compared to the same rotor when tested in isolation. The distortion of the inflow at the lower rotor compared to the relatively smooth radial variation on the upper rotor is a somewhat more direct wake interference effect-the kink in the profile at $r / R \approx 0.75$ matches very closely the position of the intersection between the blades of the lower rotor and the mean trajectory of the tip vortices from the upper rotor.

It is interesting that the inflow distribution predicted by the VTM differs quite considerably from the discontinuous inflow profile assumed 


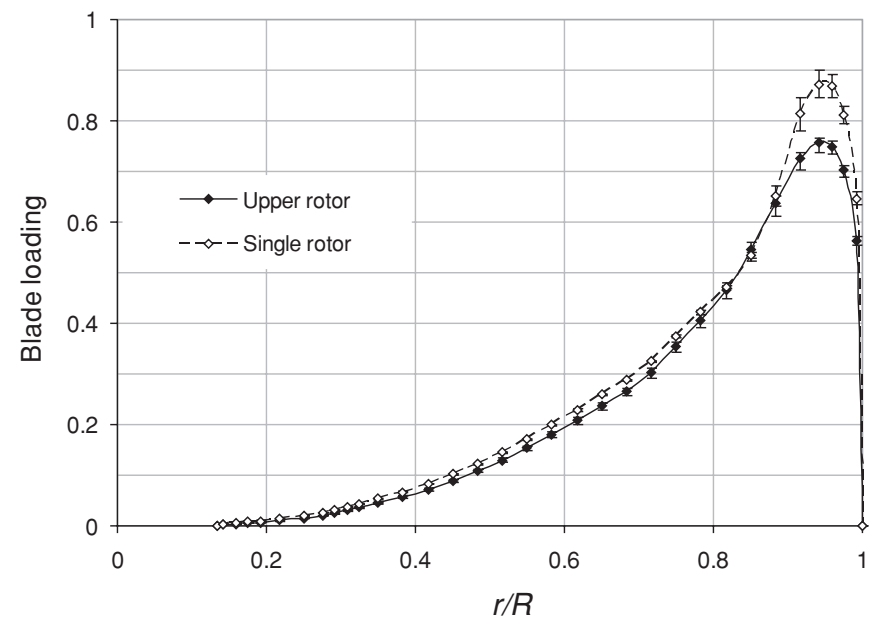

(a) Blade loading

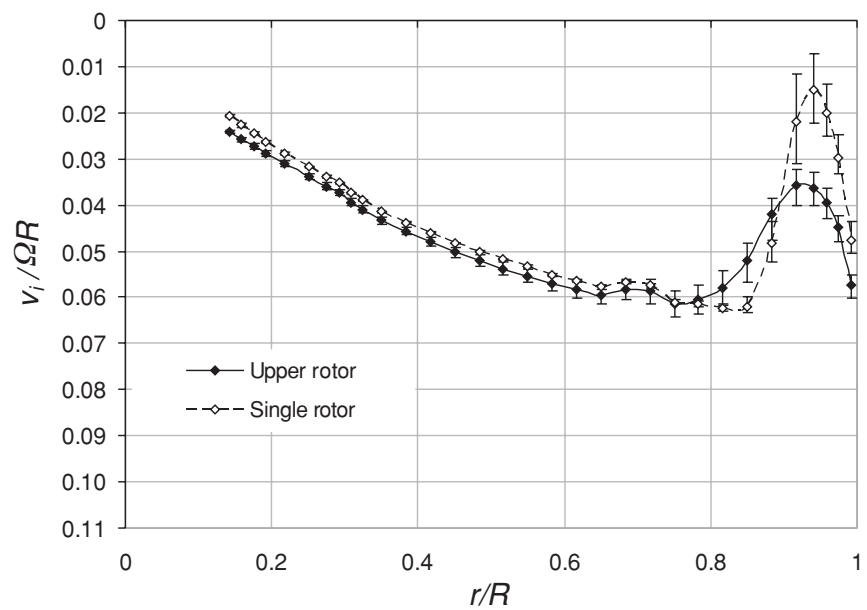

(b) Inflow

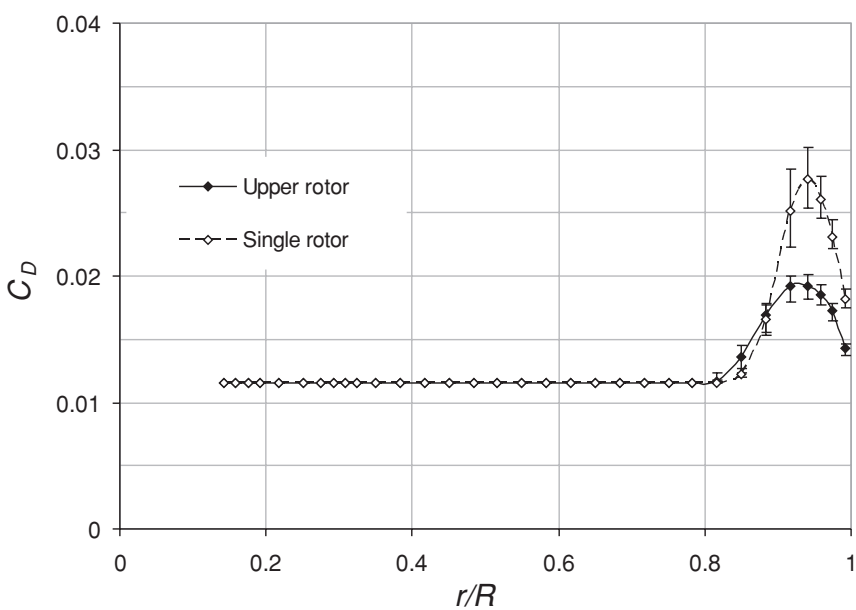

(c) Profile drag

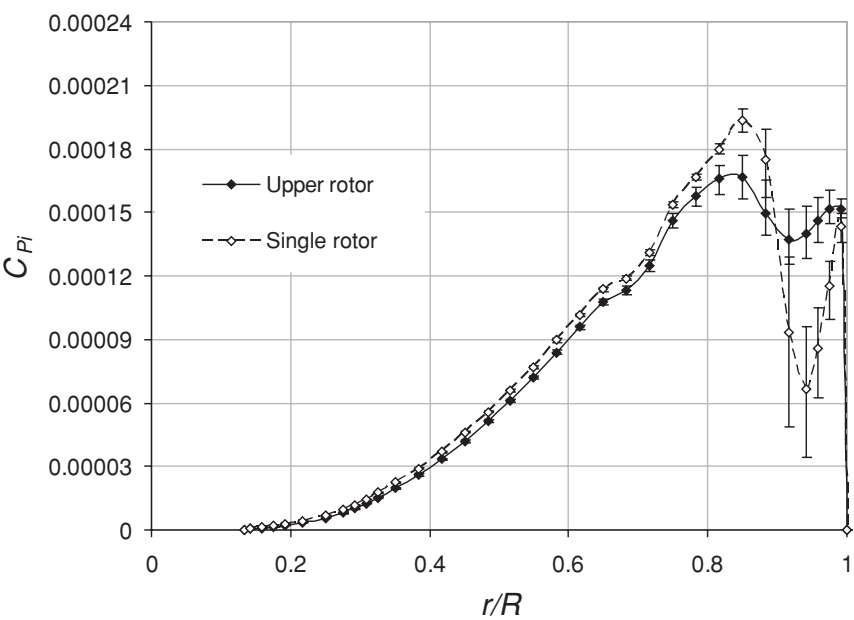

(d) Induced power

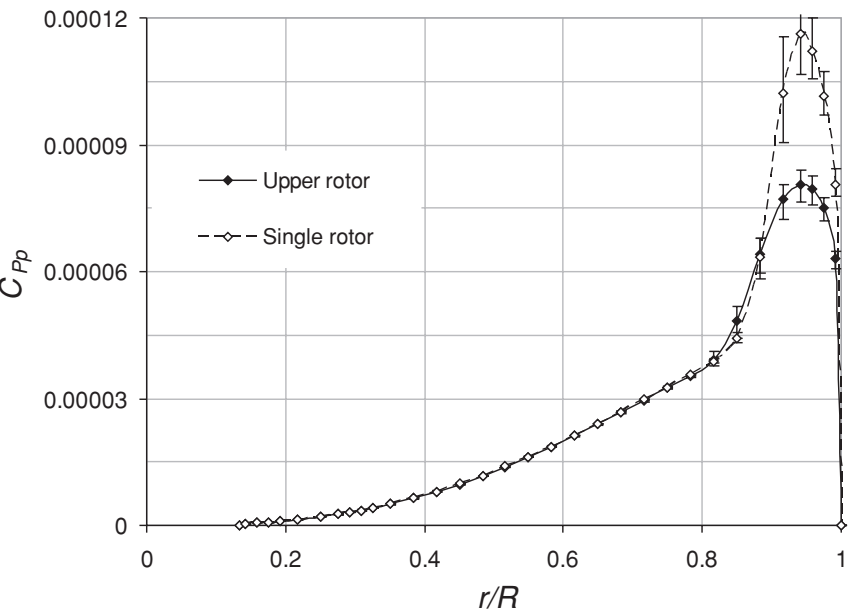

(e) Profile power

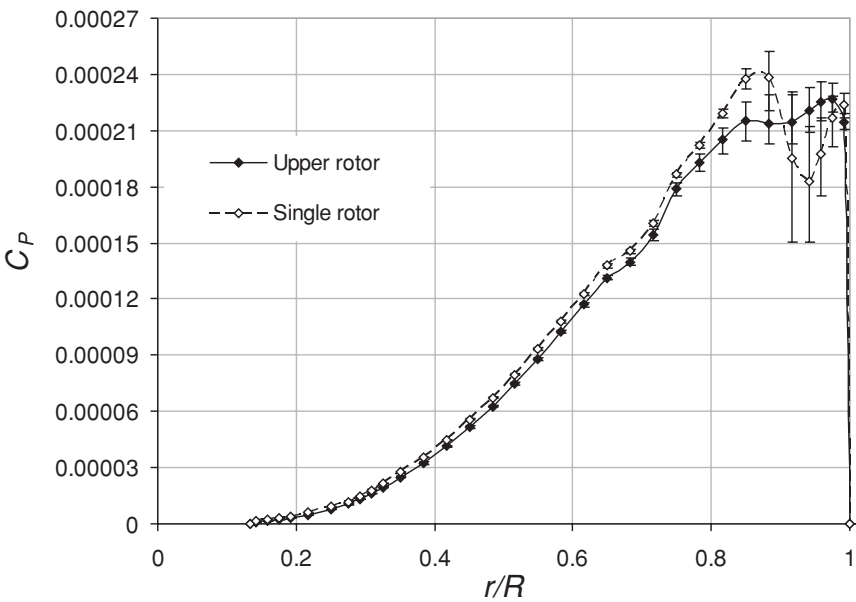

(f) Total power

Fig. 7. Radial distribution of various parameters: upper rotor of coaxial system $\left(C_{T}=0.0028\right)$ compared to one of the rotors of the coaxial system when operated in isolation at the same collective pitch setting $\left(C_{T}=0.0030\right)$.

by momentum theory (Ref. 6) or that predicted by some simpler free wake models that do not contain a representation of the inboard vortex sheet (Ref. 8).

Figure 7 compares VTM predictions of the properties of the upper rotor of Harrington's configuration when tested as part of the coaxial system and when tested in isolation at the same collective pitch setting.
Together with Fig. 6, these plots allow the behavior of each of the two rotors of the coaxial system to be compared against the behavior of a geometrically identical rotor when operated in isolation.

Figure 7(b) shows the characteristic sharp peak in the inflow distribution outboard on the rotor that results from the interaction between the blade and its tip vortex. Figure 4 shows the separation between the tip 


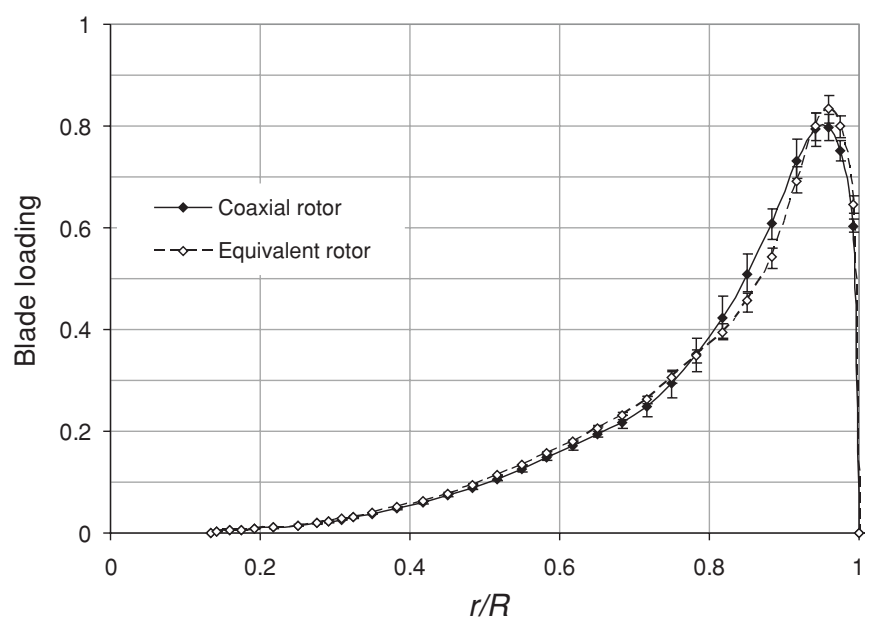

(a) Blade loading

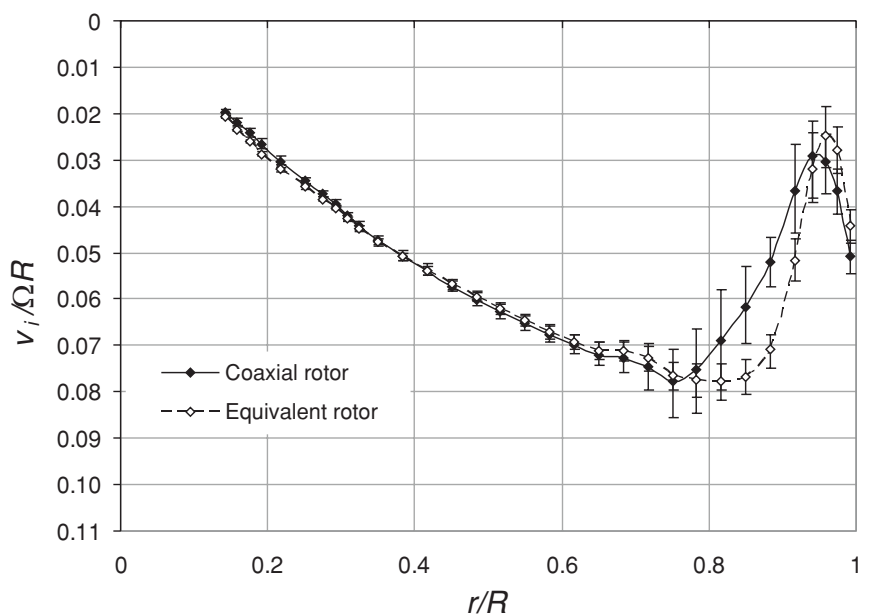

(b) Inflow

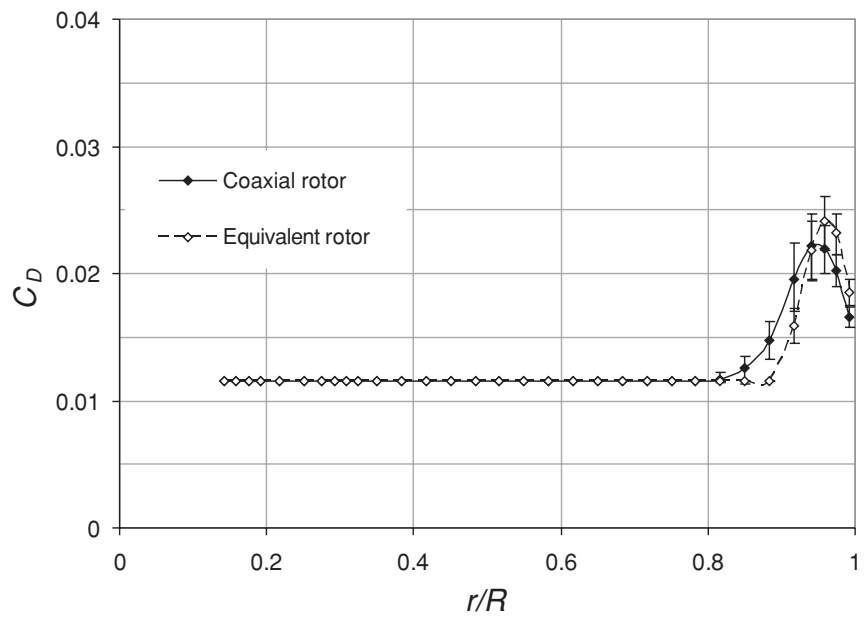

(c) Profile drag

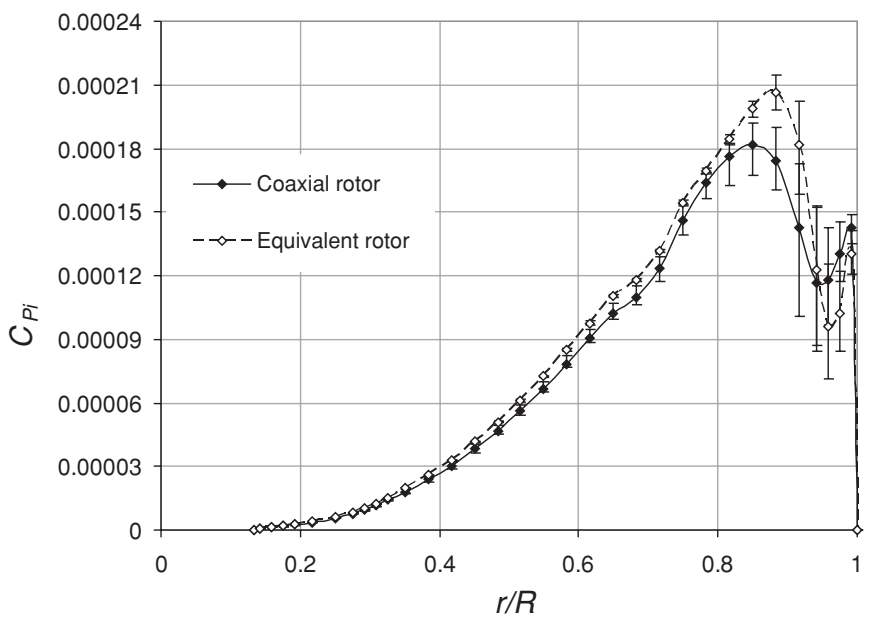

(d) Induced power

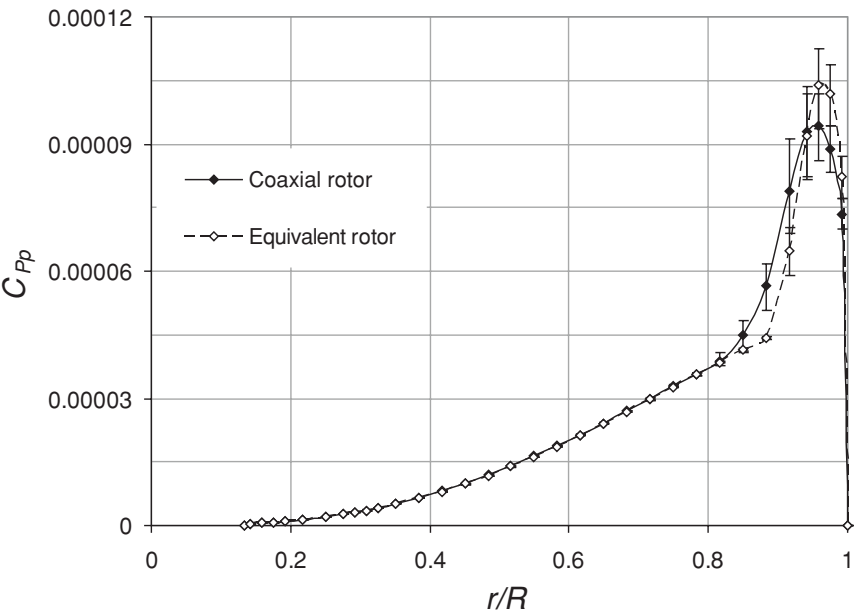

(e) Profile power

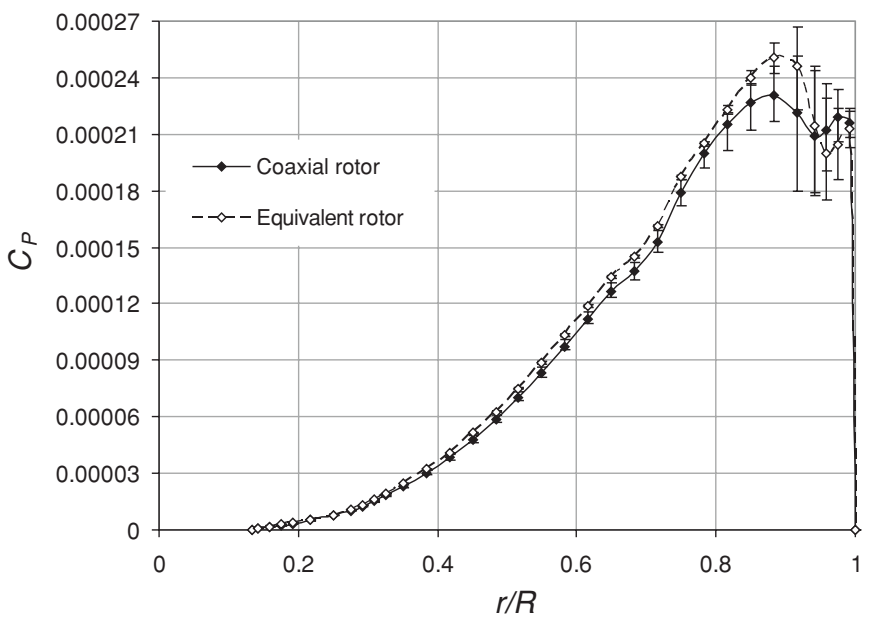

(f) Total power

Fig. 8. Radial distribution of various parameters: coaxial system (upper and lower rotors averaged) compared to the equivalent conventional rotor at the same thrust coefficient $\left(C_{T}=\mathbf{0 . 0 0 5 0}\right)$.

vortex and the blade to be somewhat greater for the rotor when part of the coaxial system than when operated in isolation, and the associated reduction in the strength of the interaction explains why this feature of the inflow distribution broadens and reduces in amplitude quite considerably when the rotor is operated as part of the coaxial system. As shown in Fig. 7(d), this change in the inflow distribution reduces considerably the peak sectional induced power consumption of the rotor when operated as part of the coaxial system rather than in isolation, but at the expense of an almost complete elimination of the sharp reduction in power consumption at the very tip of the blade. Comparing Figs. 6(d) and 7(d) shows this effect to be subtly present on the lower rotor too, but in this case the influence of the increased pitch of the tip vortices is counteracted to 


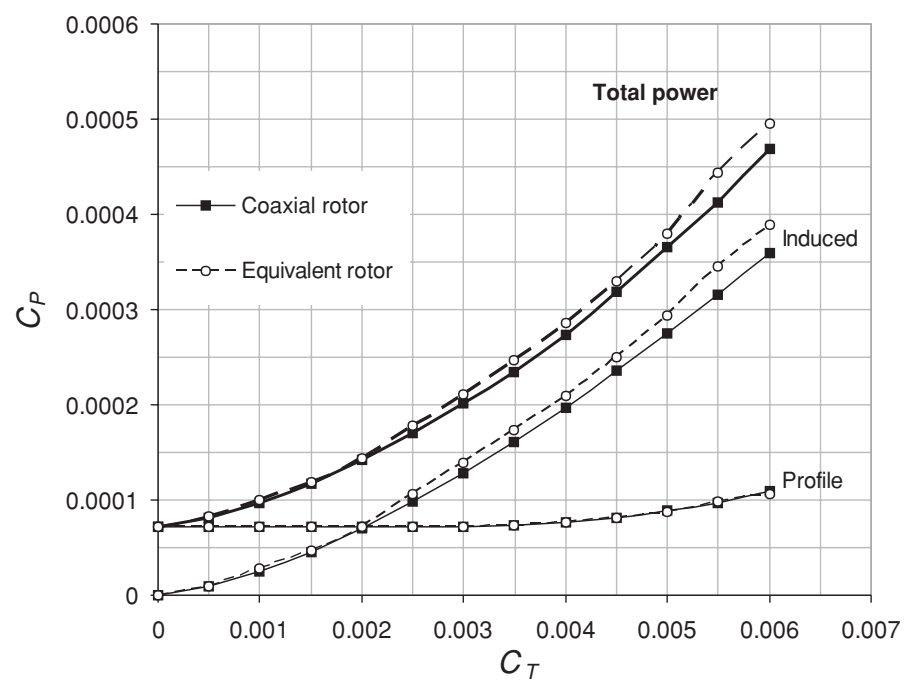

Fig. 9. Overall power and its constituents in hover.

some extent by the full immersion of this rotor in the wake of the coaxial system.

Finally, Fig. 8 compares the composite performance of Harrington's coaxial rotor (obtained by averaging the data for the upper and lower rotors of the coaxial system) against the performance of the equivalent, conventional, four-bladed rotor, allowing a direct assessment of the relative benefits of the two systems. The composite results of the various effects shown in the previous figures on the overall performance of the coaxial system relative to the equivalent conventional rotor are seen clearly in this set of figures. The spanwise variation of blade aerodynamic properties for both rotor configurations is very similar inboard on the blades-significant differences are largely confined to the outer $20 \%$ of the blade span where the differences in tip vortex geometry have the strongest effect on the distribution of aerodynamic loads on both upper and lower rotors. Most significant is the contribution of the interaction between the wake and blades, principally on the upper rotor, to a marked reduction in the induced power consumed between about $80 \%$ and $95 \%$ of the blade span of the coaxial system compared to the equivalent conventional system. This effect is the major contributor to the clear advantage, revealed in Fig. 9, that the coaxial system possesses over the equivalent conventional system in terms of its induced power consumption.

\section{Steady Forward Flight}

In level flight, cyclic pitch inputs are used to tilt the rotor tip-path plane, and hence to provide the forward component of the thrust required to overcome the drag of the system. For trimmed flight (with zero net angular acceleration), the moment generated by the aerodynamic forces on the right-hand side of the rotor system needs, at least in the timeaveraged sense, to balance that generated by the left-hand side of the system. The poor lifting efficiency of the retreating side of the disk compared to the advancing side implies that the performance of conventional rotors is often limited by the onset of blade stall on the retreating side of the rotor disk at some forward speed. In contrarotating coaxial systems with rigid rotor hubs, the aerodynamic load distributions on the left- and right-hand sides of the upper and lower rotors can be made to counteract each other's deficiencies and thus to act together to create an overall load balance between the left- and right-hand sides of the system (Ref. 9). If the coaxial rotor has an articulated hub, however, as modeled in this

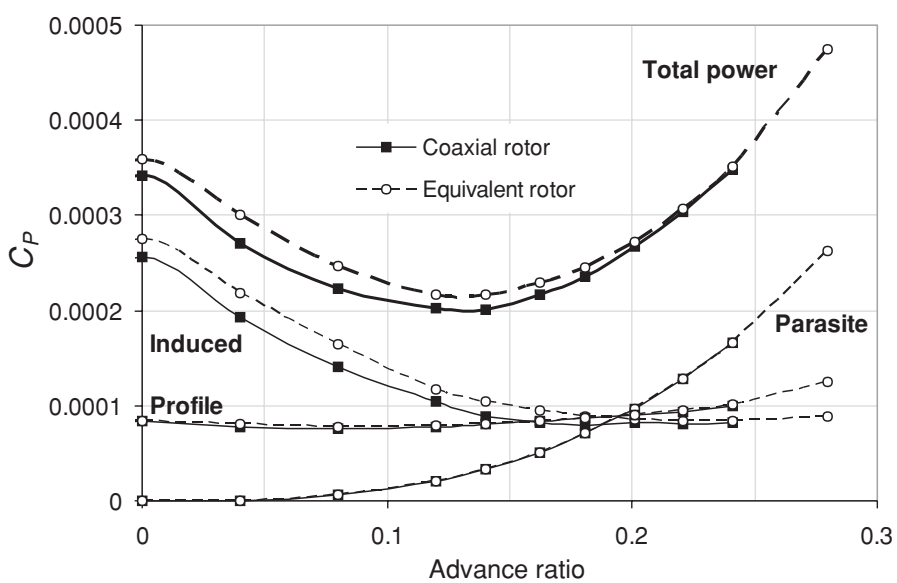

Fig. 10. Overall power and its constituents in steady level flight $\left(C_{T}=0.0048\right)$.

study, ${ }^{2}$ then the rotor flaps, in much the same manner as it does for a single rotor, to create a balance in the aerodynamic loads on each individual rotor. The natural tendency for the tip-path plane to tilt toward the advancing side on both upper and lower rotors cannot be counteracted if the cyclic control of the two rotors is mechanically interconnected, as it usually is in most practical implementations of the coaxial system. The possibility thus that the blades on the lower rotor might strike those on the upper rotor at some critical forward speed limits the minimum separation between the rotors of a coaxial system. Similarly, backward tilt of one of the rotors following blade stall on the retreating side of one of the rotors carries with it the risk of blade strike either at the front or rear of the system depending on which rotor stalls first. The next section of the paper investigates the effect of the rotor wake geometry, and the resultant interaction between the rotors, on the relative performance of coaxial and equivalent conventional rotors in steady, level flight.

\section{Forward flight performance}

VTM simulations of Dingeldein's coaxial rotor (Ref. 10) were conducted over a range of forward flight speeds. ${ }^{3}$ To represent the effects of fuselage parasite drag on the trim state of the experimental system, the forward tilt of the rotor was adjusted to produce sufficient forward force to overcome the drag corresponding to a fuselage equivalent flat plate area of 0.02 times the rotor disk area (Ref. 10). This effect was replicated in the simulations by trimming the rotor to the required force using collective pitch, aligning the shaft with the required thrust vector, and trimming out residual lateral and longitudinal forces using suitable cyclic pitch input. The simple, mechanical linkages between the upper and lower rotors of Dingeldein's coaxial system were reproduced in the simulation by applying equal cyclic pitch inputs to both upper and lower rotors. As in the experiment, differential collective pitch was used in the simulations to trim the rotor to zero net yawing moment.

Figure 10 shows the VTM-predicted variation with advance ratio of the various components of the power required by Dingeldein's coaxial rotor and its equivalent four-bladed conventional rotor. This form of presentation of the data shows the profile contribution to the power required by both systems to be almost identical at all forward flight

\footnotetext{
${ }^{2}$ Those systems with significant hub stiffness, such as Sikorsky's ABC concept, are, unfortunately, beyond the scope of this paper.

${ }^{3}$ Dingeldein's rotor was geometrically identical to Harrington's "rotor 1" that was
} simulated in the preceding section of this paper. 


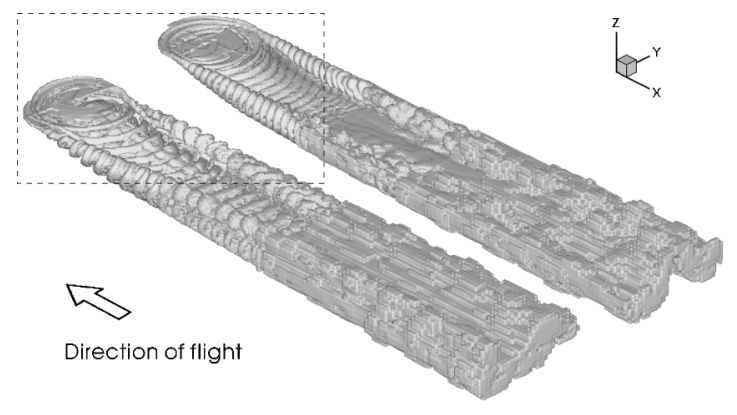

(a) Overall wake geometry

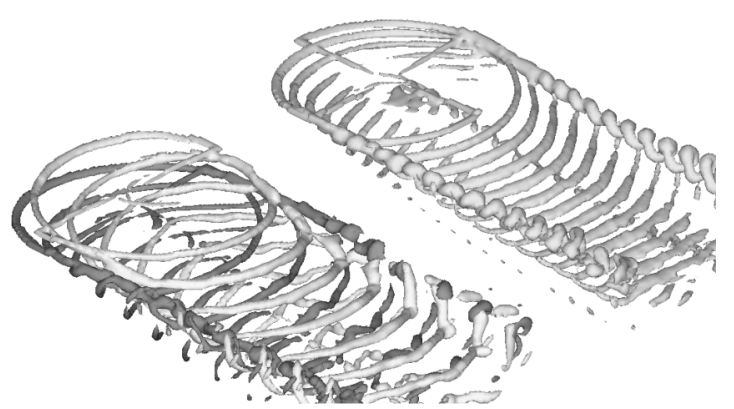

(b) Tip vortex geometry (coaxial rotor: lower rotor vortices shaded darker than upper vortices)

Fig. 11. Far- and near-field wake structure of coaxial rotor (left) and equivalent conventional rotor (right) in forward flight $(\mu=0.12)$.

speeds. The figure thus suggests that the difference in power consumption between the coaxial and equivalent systems is almost solely due to a reduction in the induced power required by the coaxial rotor compared to that required by the equivalent system. This effect is indeed seen for all advance ratios, but the major effect is in the pretransitional regime $(\mu<0.1)$ where, for instance, a reduction of about $14 \%$ in the induced power required by the coaxial rotor, compared to the equivalent rotor, is predicted at an advance ratio of 0.08 . At higher advance ratios, the induced power consumption of both rotor systems converges to similar values.

\section{Wake geometry in forward flight}

Figure 11 compares the basic features of the overall wake structures generated by Dingeldein's coaxial rotor and its equivalent four-bladed conventional rotor. In this figure, the VTM-computed wake, at an advance ratio of 0.12 and a thrust coefficient of 0.0048 , is visualized by plotting a surface in the flow on which the vorticity has constant strength. This figure illustrates well the use of nested grids within the VTM-the rotor diameter is resolved across 80 computational cells by the grid in which it is contained, but this cell size is doubled, then doubled again, downstream to reduce the resolution of the wake far from the rotor (where the wake has less direct influence on rotor performance) and hence to keep the computational requirements of the simulation within reasonable limits.

Figure 11(a) represents a surface of low enough vorticity magnitude to reveal the overall geometry of the wakes of the two systems. The gross features of the far wakes of both configurations are superficially very similar at this advance ratio: both wakes clearly show the helicoidal individual blade vortices to roll up to form a pair of large "supervortices" some distance downstream of the rotor disk. Figure 11(b) focuses on the flow near to the rotor and represents a surface of high enough vorticity magnitude to expose the detailed geometry of the tip vortices of the rotors. This figure reveals the presence of interactional aerodynamic features in the coaxial system that are not found on the conventional rotor. In particular, the complex manner in which the tip vortices from the upper and lower rotors interweave in the gap between the two rotors leads to a complicated pattern of interrotor blade-vortex interactions (BVIs) that are obviously not encountered with the conventional system. The tip vortex dynamics during the formation of the supervortices of the coaxial system is also considerably more complicated than for the conventional rotor-as can be seen, the individual tip vortices from the upper and lower rotors wind around each other, at least at this advance ratio, to form a single pair of supervortices rather than, as might be imagined, forming two distinct structures, one for each rotor.

Figures 12 and 13 show the development of the wake downstream of the two different rotor systems at various forward speeds. Figure 12 shows the overall geometry of the wake, visualized by plotting a surface in the flow on which the vorticity has constant strength, whereas Fig. 13 reveals the internal structure of the wake by showing a series of contour plots of vorticity magnitude on a longitudinal slice, containing the rotor shaft, through the wake of the system. At all advance ratios, the wake skew angles for the coaxial system and the equivalent single rotor are comparable, and the figure shows the transition of the wakes of both systems from their tubular, skewed but still essentially hover-like structure at low advance ratio to a flattened, airplane-like geometry at higher advance ratio. This transition is counteracted at high advance ratio, to some extent, by the forward tilt of the rotor that acts to maintain the tubular structure of the wake. At low advance ratio, the wake does not roll up to form the stable, well-structured supervortices seen at higher forward speeds. Instead, the individual wakes from each of the blades interact in an unsteady process that involves the co-orbiting and eventual pairing of nearby tip vortices to form larger vortical structures further downstream in the wake, in much the same fashion as in hover. Interestingly, the coaxial rotor appears to produce a more structured wake downstream of the system than the equivalent conventional rotor does at the same advance ratio. This is particularly noticeable at forward speeds below $\mu=0.08$. In Refs. 11 and 12, it was argued that the rate of development of perturbations to the ordered, helicoidal structure of the wake should be greater the more closely spaced the individual vortices within the wake. Even though both rotors have the same overall number of blades, the advantage, in terms of wake stability, of the coaxial rotor in producing two, albeit closely spaced, streams of tip vortices with effectively twice the spacing between vortices of the equivalent rotor, is clearly evident. Whether this effect turns out to have a significant practical benefit, for instance in terms of its influence on tail-shake or other types of rotor-fuselage interference, remains to be shown.

Figure 14 illustrates the corresponding changes in character of the interaction between the upper and lower rotors of the coaxial system as the forward speed of the rotor is increased. In this sequence of diagrams, the spanwise distribution of inflow experienced by a single blade, during a single rotor revolution, is plotted as a polar function of blade azimuth. Figure 5 shows the almost axially symmetric distribution of inflow on both rotors when in hover, but this symmetry is destroyed in forward flight as a result of the skew of the rotor wake. At the lowest forward speeds, a prominent ridge is created in the inflow experienced by the blades of both the upper and the lower rotor as they traverse the forward half of their respective disks. This, and a series of weaker ridges further aft on the disk, results from intrarotor BVI where the blades interact with the tip vortices trailed from blades on the same rotor. These features weaken as the forward speed of the system is increased, and the rotors tilt forward to provide an increasing propulsive component of thrust. Figure 13 shows that the tip vortices from the upper rotor pass through 

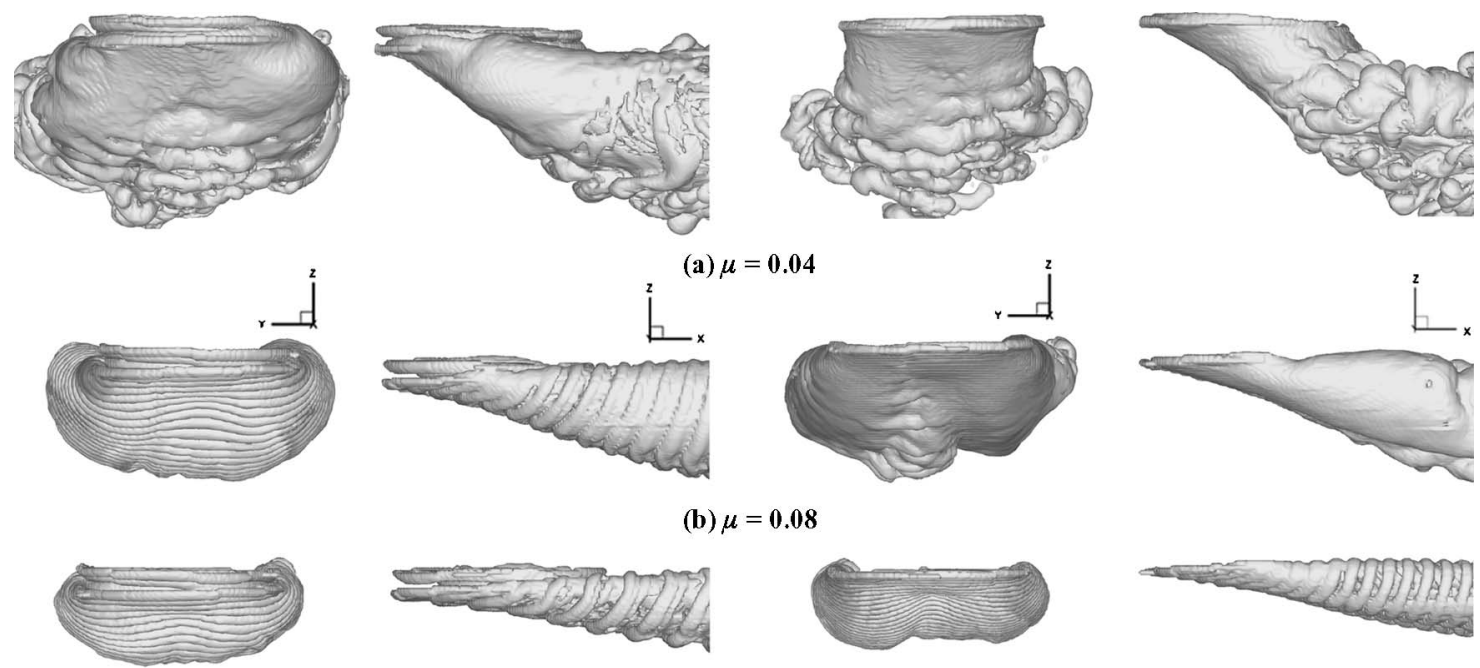

(c) $\mu=0.12$
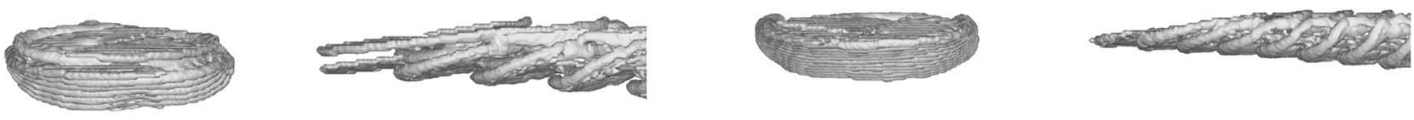

(d) $\mu=0.24$

Fig. 12. Wake geometry in steady, level flight (left: coaxial rotor; right: equivalent conventional rotor).

the plane of the lower rotor at all forward speeds. The interaction of these vortices with the lower rotor is seen in Fig. 14 as a second series of ridges in the inflow on the advancing side and rear of the lower disk that are interdigitated with the intrarotor BVIs. Compared to the intrarotor BVIs, these interrotor BVIs are persistent, and, as forward speed is increased, the primary interrotor BVI becomes the predominant feature of the inflow on the advancing side of the lower rotor. This strong interrotor interaction, which is absent in the case of a single rotor, may contribute as a significant source of noise.

Figure 15 shows how the inflow at high advance ratio results in a load distribution on the lower rotor that, apart from some features on the advancing side, bears a more than superficial resemblance to the mirror
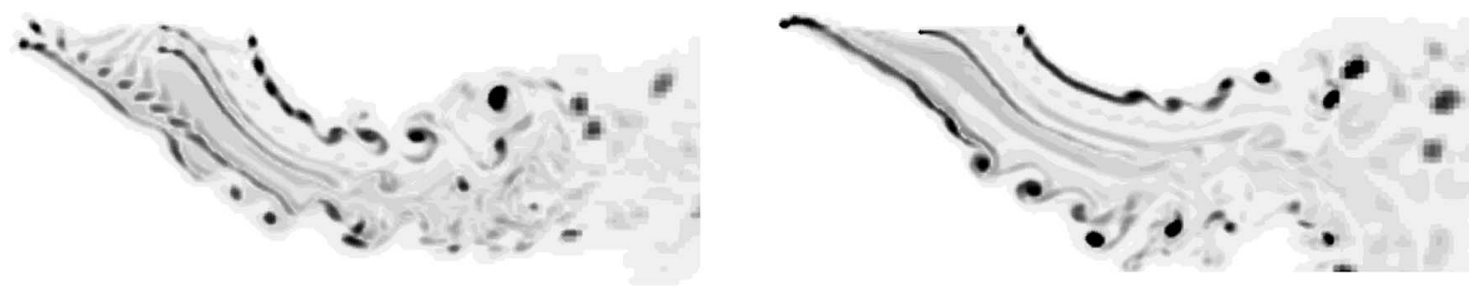

(a) $\mu=0.04$
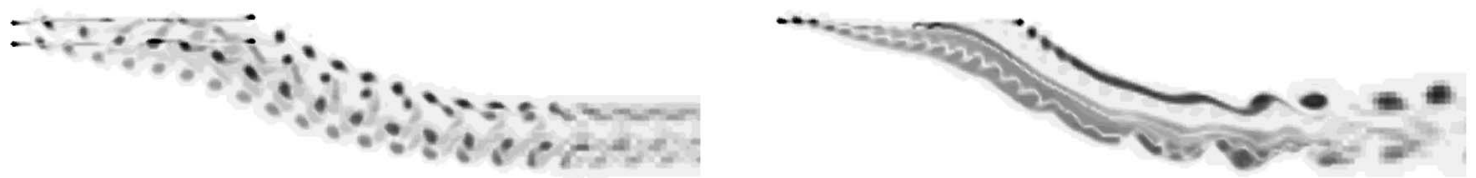

(b) $\mu=0.08$
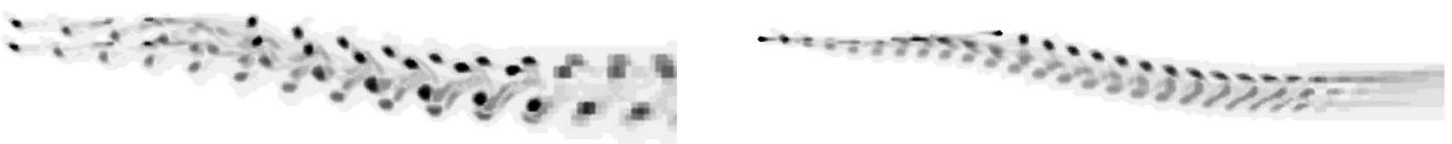

(c) $\mu=0.12$
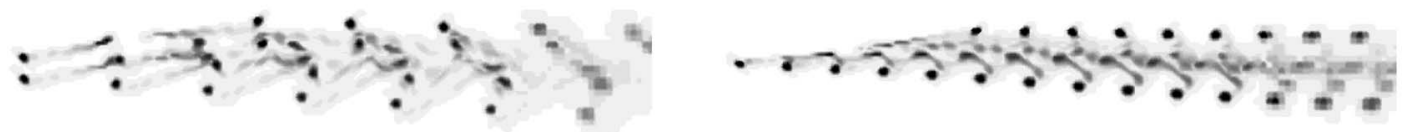

(d) $\mu=0.24$

Fig. 13. Internal wake structure in steady, level flight (left: coaxial rotor; right: equivalent conventional rotor). 

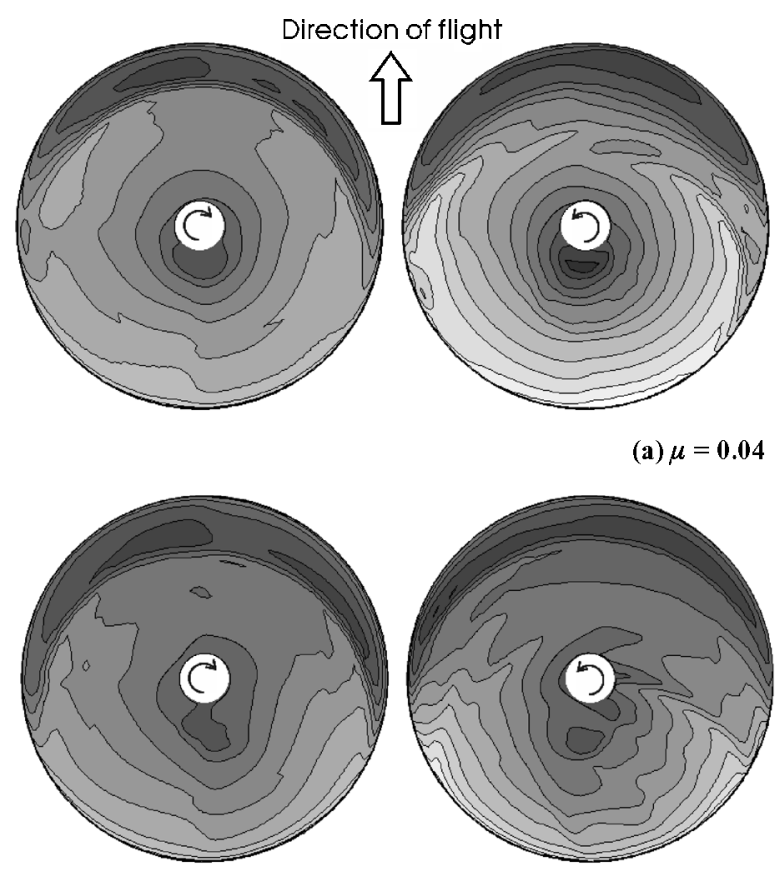

(b) $\mu=0.08$
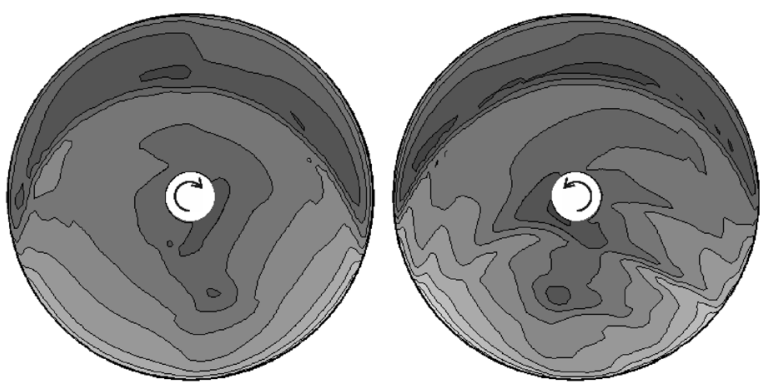

(c) $\mu=0.12$
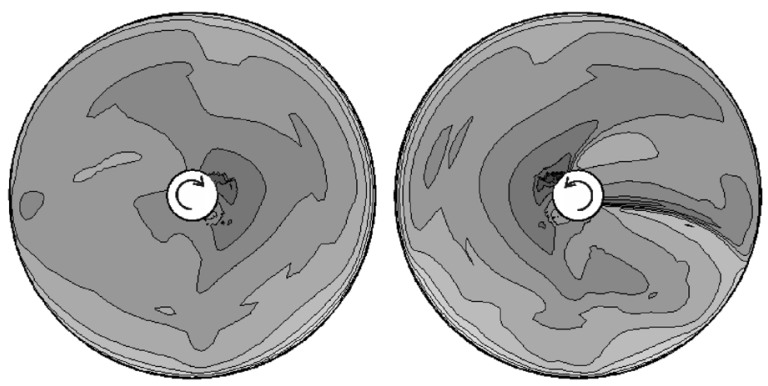

(d) $\mu=0.24$
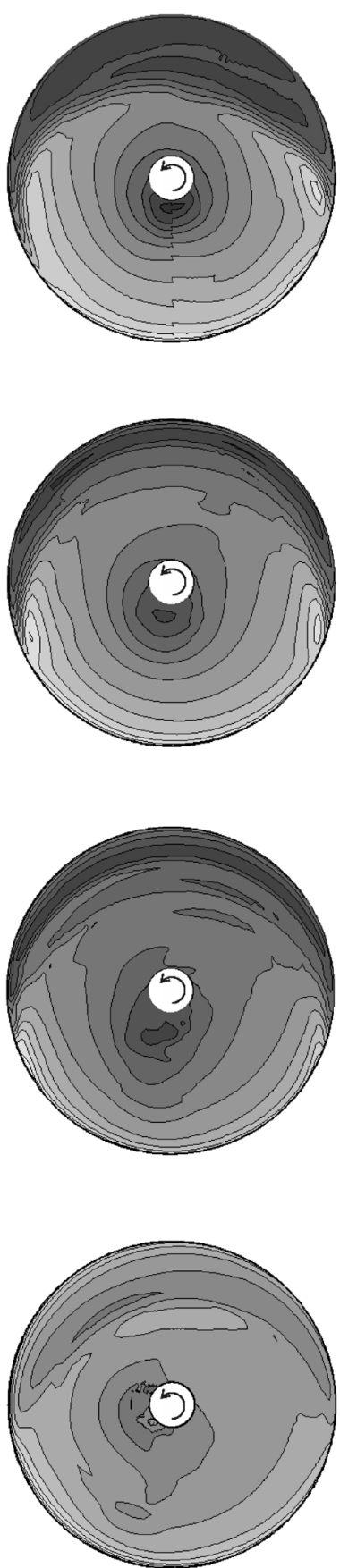

$v_{i} / \Omega R$

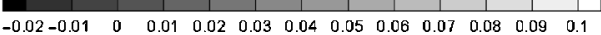

Fig. 14. Spanwise inflow variation experienced by a single blade during one revolution at various advance ratios (far left: upper rotor of coaxial system; left: lower rotor of coaxial system; right: equivalent conventional rotor).

image of the loading on the upper rotor. This figure thus illustrates well how each individual rotor of a coaxial system with articulated hubs maintains its own individual load balance through blade flapping. The effect of forward speed on the control inputs that are required to trim the rotor are shown in Fig. 16, whereas the adverse consequences of mechanical interlink between the cyclic controls of the two rotors are revealed in Fig. 17. In this figure, the separation between the tips of the blades of the upper and lower rotors is plotted against advance ratio at each of the four azimuthal positions (with respect to the lower rotor) at which blade strike might possibly occur in a twin, two-bladed coaxial system (i.e., those azimuths at which the blades from the lower rotor pass directly underneath the blades of the upper rotor). Recalling that the separation between the upper and lower hubs is $0.19 R$, the tip clearance at the front and back of the rotor remains more or less constant throughout 


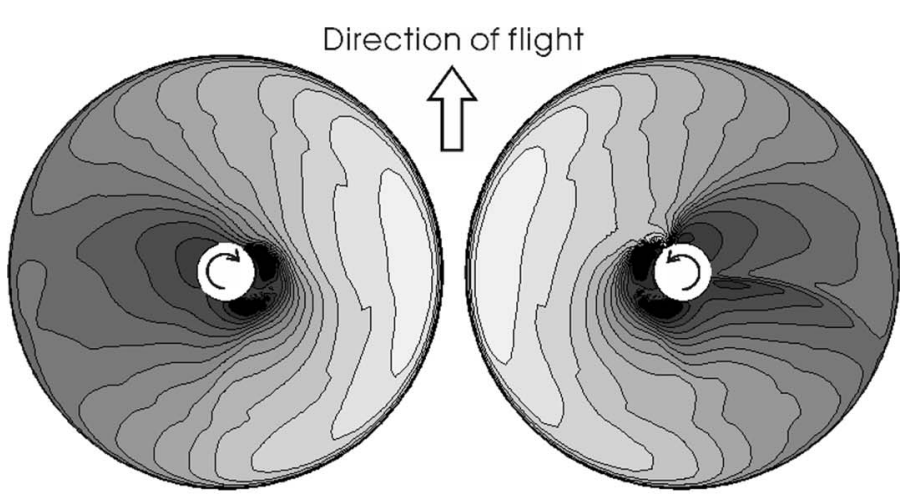

(a) Coaxial rotor (left: upper rotor; right: lower rotor)

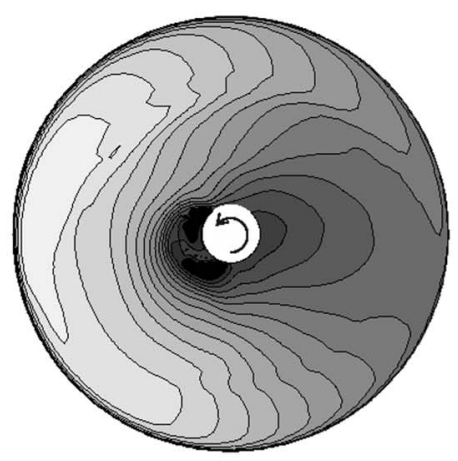

(b) Equivalent conventional rotor

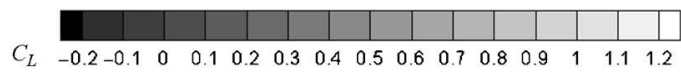

Fig. 15. Spanwise variation of lift coefficient experienced by a single blade during one revolution at advance ratio 0.24 .

the flight envelope. The mechanical interlink between the two rotors does not allow independent control of the lateral cyclic inputs to the rotors, though, and the resultant lateral flapping toward the advancing side of both rotors reduces significantly the lateral separation between the two rotors at $270^{\circ}$ azimuth (defined with respect to the lower rotor). This effect is particularly marked at pretransitional advance ratios, although, for the rotor as operated, there was never any danger of blade strike. Similar variations of blade clearance with advance ratio are observed in flight tests of full-scale coaxial systems with articulated rotors and mechanical cyclic interlink (Ref. 13). Note that the computational results extend to forward speeds beyond the maximum advance ratio that was tested in Dingeldein's experiments. The onset of blade stall on the retreating sides of both disks results in extreme longitudinal blade flapping. In this case, a sudden reduction in clearance between the rotors at high advance ratio is precipitated by the dynamics of the upper rotor which stalls earlier and more abruptly than the lower rotor yielding the definite possibility of blade strike at the back of the rotor beyond an advance ratio of approximately 0.33 .

\section{Maneuvering Flight}

Proponents of the coaxial rotor system claim that higher levels of maneuverability can be achieved than with conventional systems because of several practical engineering advantages of the coaxial configuration: for instance, the increased control moments because of the length of the rotor mast that is required for adequate rotor separation, the low moments of inertia of the airframe, since generally this can be made quite compact because a tail rotor is not required, and so on (Ref. 13).

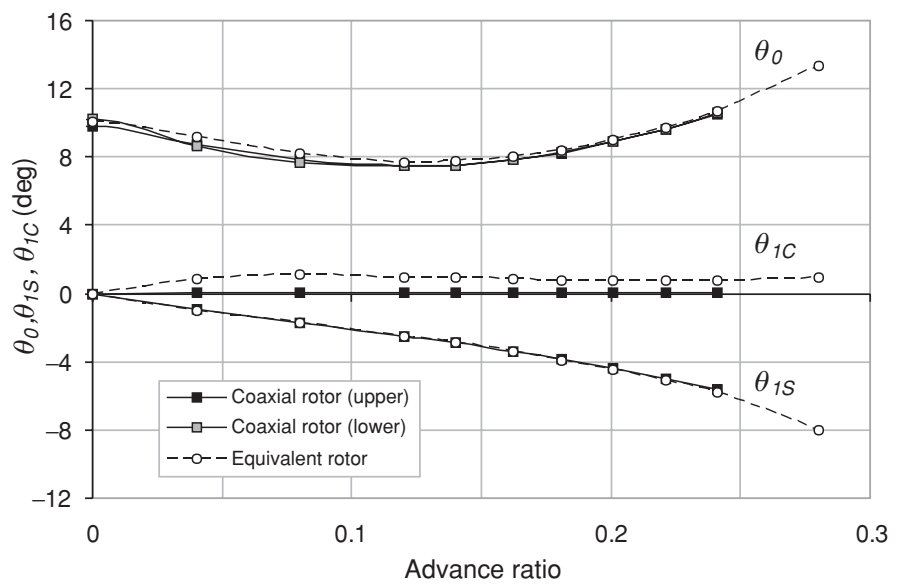

Fig. 16. Control angles required for trim in steady level flight $\left(C_{T}=0.0048\right)$.

This section of the paper explores the relative advantages and disadvantages of the coaxial rotor configuration in maneuvering flight that arise from the particular aerodynamic characteristics of the system. VTM calculations of the performance of a coaxial system in a coordinated turn are presented, and compared against similar calculations of the performance of an equivalent conventional rotor with equal solidity and the same number and design of blades as the coaxial system. For consistency with the earlier sections of this paper, Dingeldein's twin, two-bladed coaxial rotor was used as the basis for comparison.

In addition to the forward component of force required to overcome the fuselage parasite drag, in a trimmed, coordinated, level turn the rotor is required to produce a lateral force to provide the necessary centripetal acceleration. For a rotor with no hinge offset, the equilibrium lateral shaft inclination $\gamma=\cos ^{-1}(1 / n)$, to first approximation, where the load factor $n$ is defined as the ratio of the rotor thrust required during the maneuver to the rotor thrust during steady level flight. In a coordinated turn, there is no lateral component of the rotor thrust with respect to the rotor shaft axis, and the force required to accelerate the rotor in the turn comes solely from the lateral inclination of the rotor disk.

As an example, for a steady level turn with $n=1.5$ and $\mu=0.12$, the required lateral shaft inclination is $48.2^{\circ}$ and the radius of the resulting turn is approximately $7 R$. The wake structure generated by the coaxial rotor in such a turn (with $C_{T}=0.0072$ when $n=1.50$ ) is shown in Fig. 18 - the rotor, in a steep left-hand bank, can be seen on the left-hand side of the image. The distortion to the wake geometry as a result of the maneuver is clearly evident.

\section{Turn performance}

Significant savings in computational time can be achieved by calculating the turn performance of a rotor system in a simulation of the real-world dynamic maneuver known as a "wind-up turn." In this maneuver, the load factor on the rotor is increased steadily with time, rather than being held constant. If the rate of increase of the load factor on the system is kept small enough, then the results should be the same as those obtained in a series of individual tests in turns at constant load factor. Figure 19 shows the variation of the VTM-predicted power consumption with increasing load factor, for both the coaxial system and its equivalent conventional rotor, in a coordinated, level wind-up turn to the left. The advance ratio was held constant at 0.12 throughout the simulation, and the thrust was set so that, in steady level flight, $C_{T}=0.0048$. As in the forward flight simulations of the coaxial rotor, a simple mechanical 


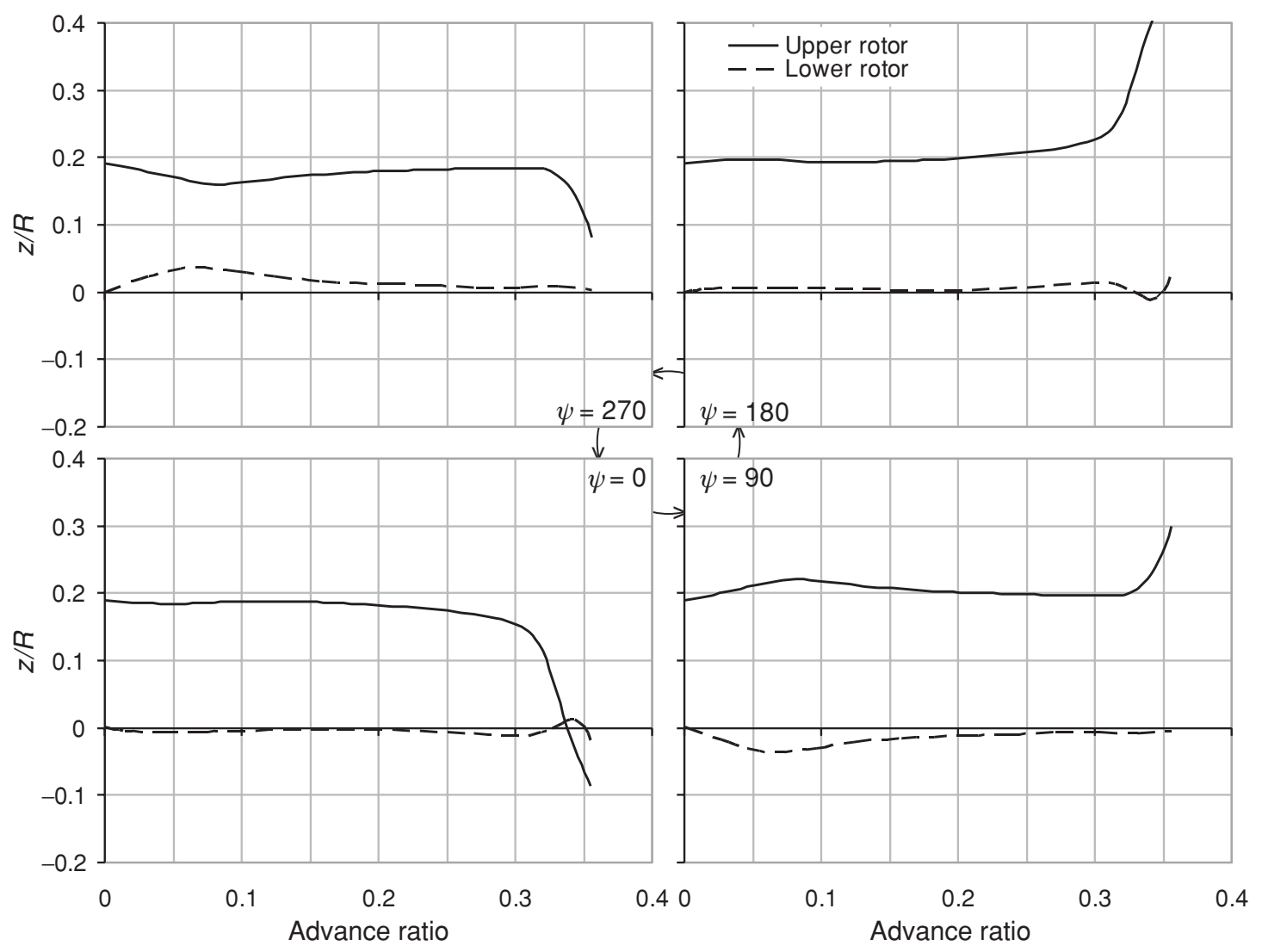

Fig. 17. Vertical clearance between the blade tips of the coaxial rotor in steady, level flight $\left(C_{T}=0.0048\right.$; azimuth defined with respect to the lower rotor).

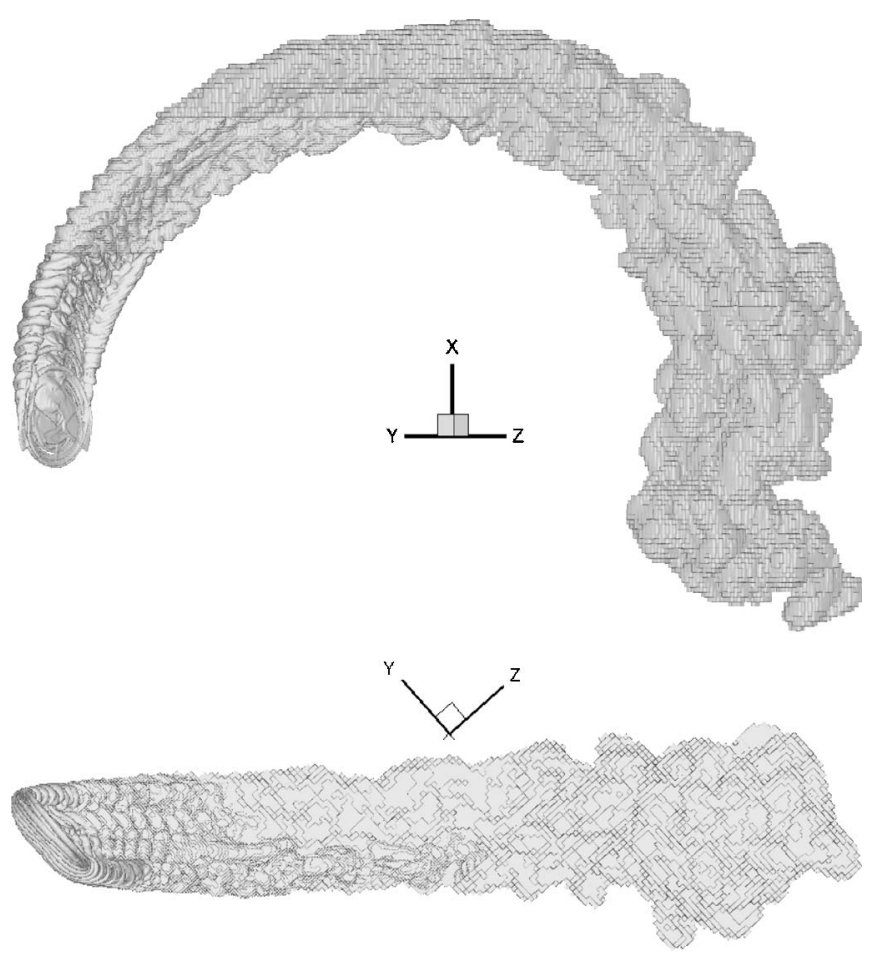

Fig. 18. Wake of a coaxial rotor in a coordinated, level, left-hand turn at load factor 1.50 (top: top view; bottom: front view).

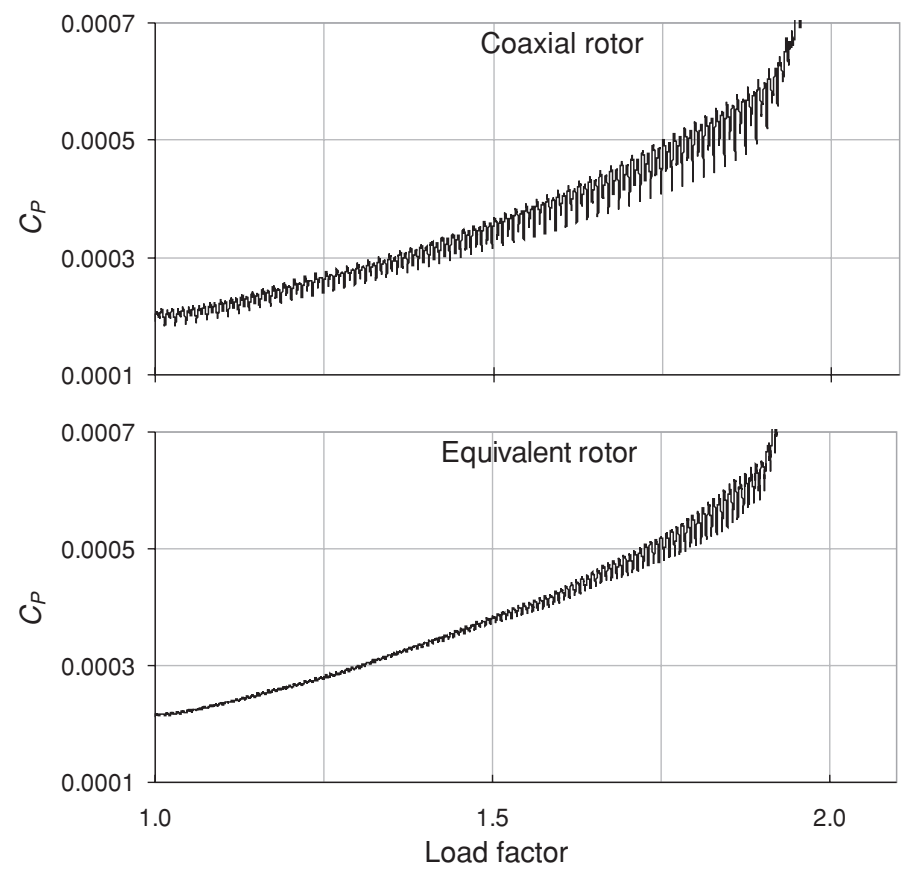

Fig. 19. Total power required by coaxial and equivalent systems in a level, wind-up turn. 


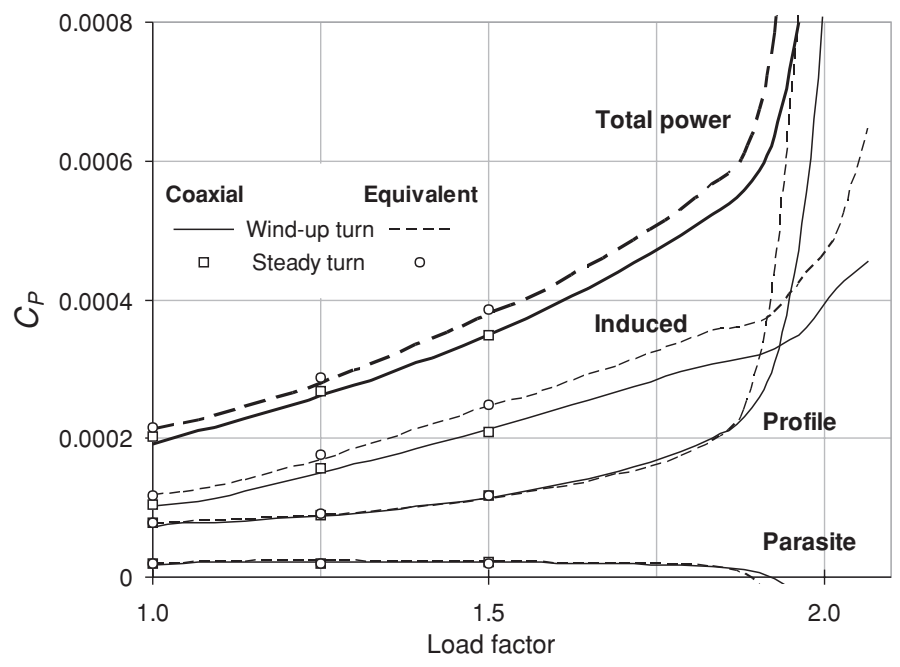

Fig. 20. Total power and its constituents in a level, wind-up turn (with high-frequency oscillations removed).

cyclic control system was modeled by applying the same cyclic control inputs to both rotors and using differential collective to give zero net yawing moment about its shaft. ${ }^{4}$

Figure 19 shows the unfiltered time history of the overall power consumed by both rotor systems during the wind-up turn. Figure 20 shows a breakdown of the power into parasite, profile, and induced components after filtering to remove all harmonics in the unsteady data at greater than the blade passing frequency. Results from simulations of steady turns at $n=1.25$ and 1.5 are also shown to confirm the independence of the results of the unsteady simulation from the effects of the rate of increase of the load factor on the rotor. Although all the data presented here are for a left-hand turn, simulations of steady performance in the equivalent right-hand turn, most interestingly, show a slight increase in power requirement (of about $1.5 \%$ for the coaxial rotor and $2.1 \%$ for the equivalent system at a load factor of 1.5 ). The divergence in power that is seen at a load factor of approximately 1.7 is principally confined to the profile component of the power consumption of both rotors and indicates the onset of blade stall. The slightly earlier rise in the profile power consumption of the conventional rotor suggests that the coaxial system possesses a small (approximately $0.1 \mathrm{~g}$ ) advantage over the equivalent conventional rotor in terms of absolute maximum maneuver performance, but the limitations of the model used for the profile drag of the rotor sections need to be borne in mind before any generalizations can be made to the real world in this respect. On the other hand, the simulations also show a consistent advantage of the coaxial configuration over the conventional system in terms of the power required for the turn (of approximately $8 \%$ compared to the conventional system) at all prestall load factors. The almost complete confinement of this effect to the induced power predictions of the model suggests that this benefit may indeed be realized in the real-world system. Comparison of the unfiltered response of the two systems, as shown in Fig. 19, suggests, though, that the improved performance of the coaxial system may come at the cost of significantly higher rotor vibration. A detailed analysis of the high-frequency dynamics of the system is beyond the scope of the present work, however.

\footnotetext{
${ }^{4}$ This is, of course, somewhat of an approximation, since, for instance, it yields a constant-rate turn only in the absence of fuselage aerodynamic moments.
}

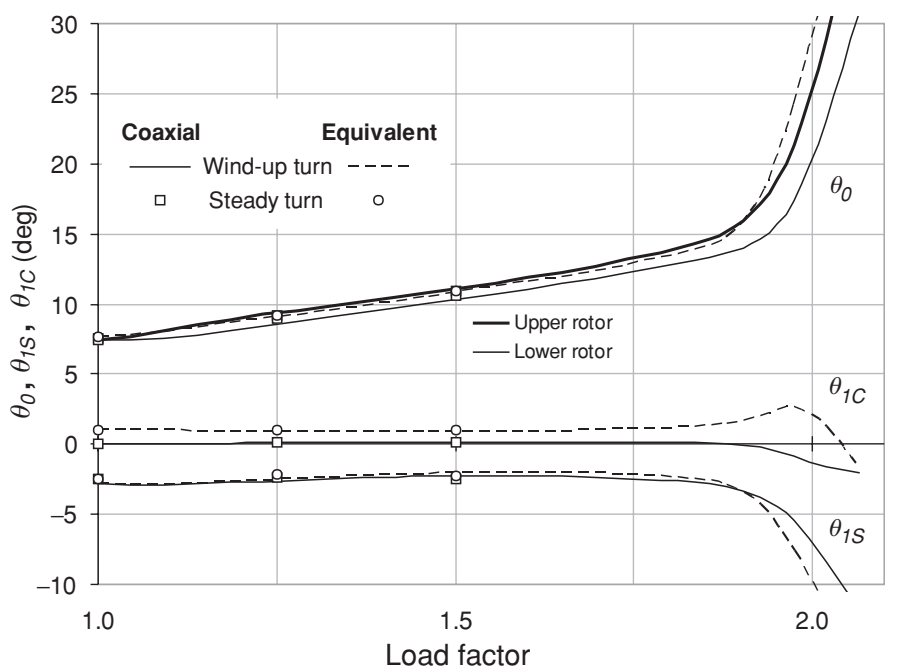

Fig. 21. Control angles required in a level, wind-up turn.

The predicted variation of the control inputs with load factor is shown in Fig. 21, and, as in the level, forward flight simulations, reveals the principal difference between the two rotor configurations to be in the lateral cyclic pitch required to trim the systems in the turn. Figure 22 shows the clearance between the rotors during the wind-up turn to be fairly independent of load factor, up until the onset of stall, beyond which the separation between the rotors decreases rapidly and blade strike becomes a distinct possibility. Unlike in the forward flight case, where stall-induced blade strike occurred at the rear of the rotor, the modification of the loading distribution on the rotors at stall due to the angular velocity of the system in conjunction with the dynamics of the rotor in the accelerated reference frame of the turn drives the position of initial blade strike to the left of the rotor (remembering that the results presented in this section are for simulations of a left-hand turn).

\section{Wake geometry in a steady turn}

Figure 23 contrasts the development of the inflow distribution on the coaxial system and the conventional rotor as the load factor in the turn is increased. These diagrams, as in the forward flight comparisons, show the spanwise variation of inflow experienced by one of the blades of the rotor during a single revolution, and are best interpreted in conjunction with Figs. 24 and 25, which contrast the geometries of the wakes of the two systems. To represent properly the effects of a steady turn on the curvature of the wake, the images in these figures are all taken from the simulations of steady left-hand turns at constant load factor, rather than from the simulation of the wind-up turn. If anything, the diagrams reveal the rather subtle nature of the shifts in loading distribution that accompany any change in load factor. On the conventional rotor, a slight increase in blade-wake interaction at both $70^{\circ}$ and $290^{\circ}$ azimuth accompanies the lifting of the trajectory of the supervortices relative to the disk plane as the curvature of the wake increases with load factor. As the load factor on the coaxial rotor is increased, the rearward of the two interrotor BVIs on the advancing side of the lower rotor decreases in strength relative to the forward BVI, and the influence of the root vortices on the loading on the rear of both rotors increases. At the same time, another interrotor BVI on the retreating side of the lower rotor just aft of the primary intrarotor BVI becomes steadily more prominent. Little obvious change in the geometry of the wake accompanies these changes in the pattern of BVIs 

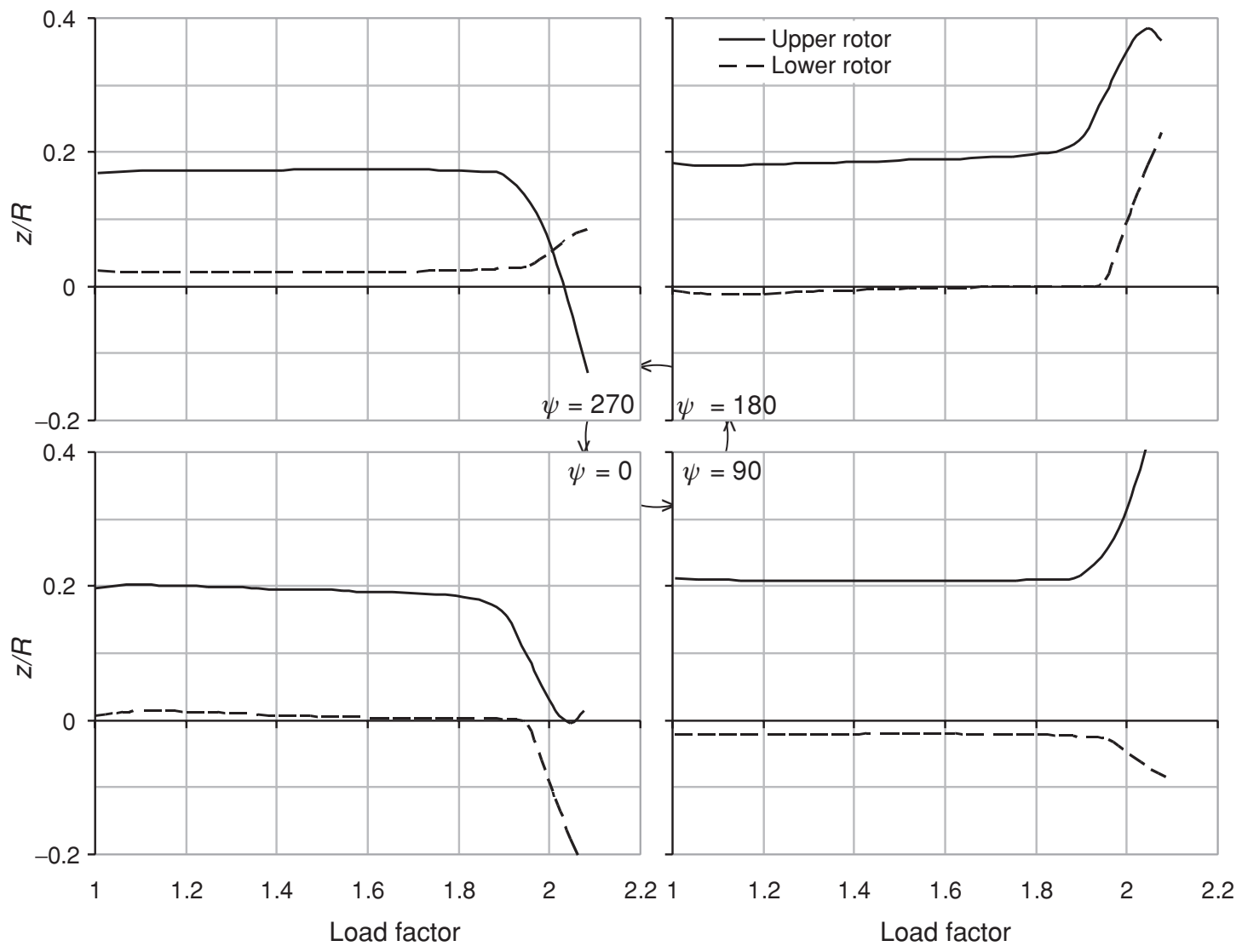

Fig. 22. Evolution of the vertical clearance between the blade tips of the coaxial rotor during a wind-up turn (azimuth defined with respect to the lower rotor).

on the rotors, but the lifting of the wake into the plane of the rotors as a result of the curved trajectory of the system is clearly apparent in Figs. 24 and 25 .

\section{Aerodynamic origin of power differences}

The analysis of hover, forward flight, and steady level turns shows the benefit of the coaxial rotor to lie in its reduced consumption of induced power compared to the equivalent conventional rotor. Much insight into the relative performance of the two systems can be obtained by localizing this benefit to specific features of the aerodynamic loading on the rotors. Figure 26 uses the VTM-predicted spanwise distribution of sectional induced power on the rotor blades to achieve this aim. In the far left-hand images, the sectional induced power distribution on a second type of coaxial-equivalent rotor (that essentially neglects the vertical separation between the rotors) is defined by averaging the contributions (at the same azimuth, defined with respect to the lower rotor) to the sectional induced power from blades on the upper and lower rotors of the coaxial system. This composite distribution can be contrasted with the radial variation of sectional induced power on the equivalent conventional rotor (defined as in previous sections of the paper) shown in the middle images. The difference between the distributions of sectional induced power on these two, differently defined equivalent rotors is shown at right and provides a measure of the relative performance of the coaxial and conventional equivalent systems that is localized to specific features in the aerodynamic loading distribution on the two rotors. Light regions of the figures correspond to parts of the rotor disk where the coaxial rotor consumes less power than the conventional system and vice versa. As is to be expected from the relatively small difference in power consumption between the two systems, the features in the aerodynamic loading that yield the differences in overall induced power consumption are relatively subtle.

It appears that the relative merits of the two systems are closely tied to the position and strength of the various BVIs on the disk plane. In hover, the equivalent rotor benefits compared to the coaxial system from the slight reduction in induced power that is associated with a small reduction in the downwash that is induced on the very outboard part of the disk by a slightly stronger tip-vortex interaction. A similar reduction inboard is associated with a similar difference in the character of the interaction of the blades with the root vortex. These benefits are outweighed on the whole, though, by the presence of a relatively broad band of lower induced power consumption just inboard of the tip of the coaxial system. Comparing Figs. 7(d) and 8(d) shows that this effect results from a favorable redistribution of the loading slightly inboard of the tip of the upper rotor, and is most likely due to the amelioration of the blade-vortex interaction on this rotor as a result of the increased axial convection rate of the tip vortices of the coaxial system compared to the equivalent rotor.

The interrotor BVIs that are such a prominent feature of the inflow distributions shown in Fig. 14 have little effect on the relative apportionment of induced power between the two systems in forward flight since they primarily affect the loading inboard on the rotor where their contribution to the power required by the system is somewhat diminished. The conventional rotor gains some advantage over the coaxial system from 

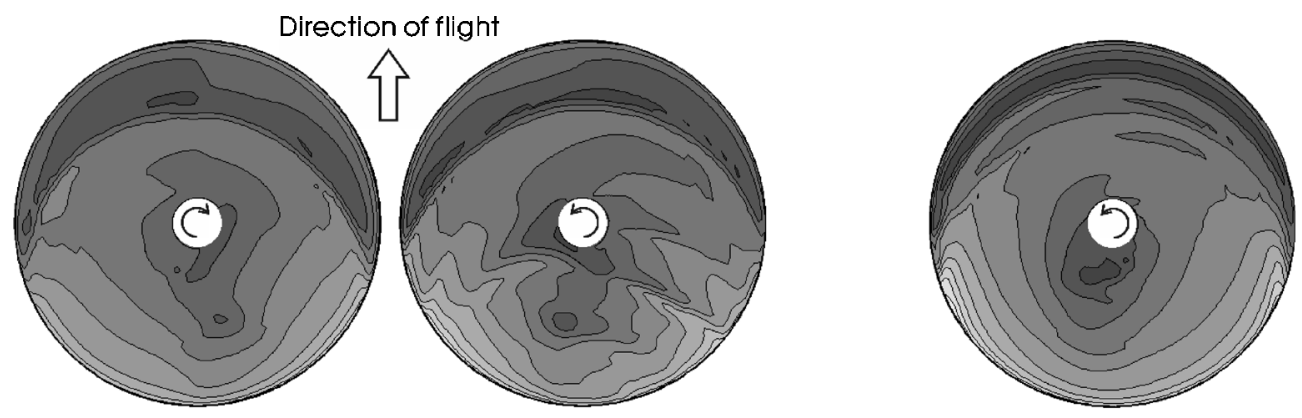

(a) $n=1.00$
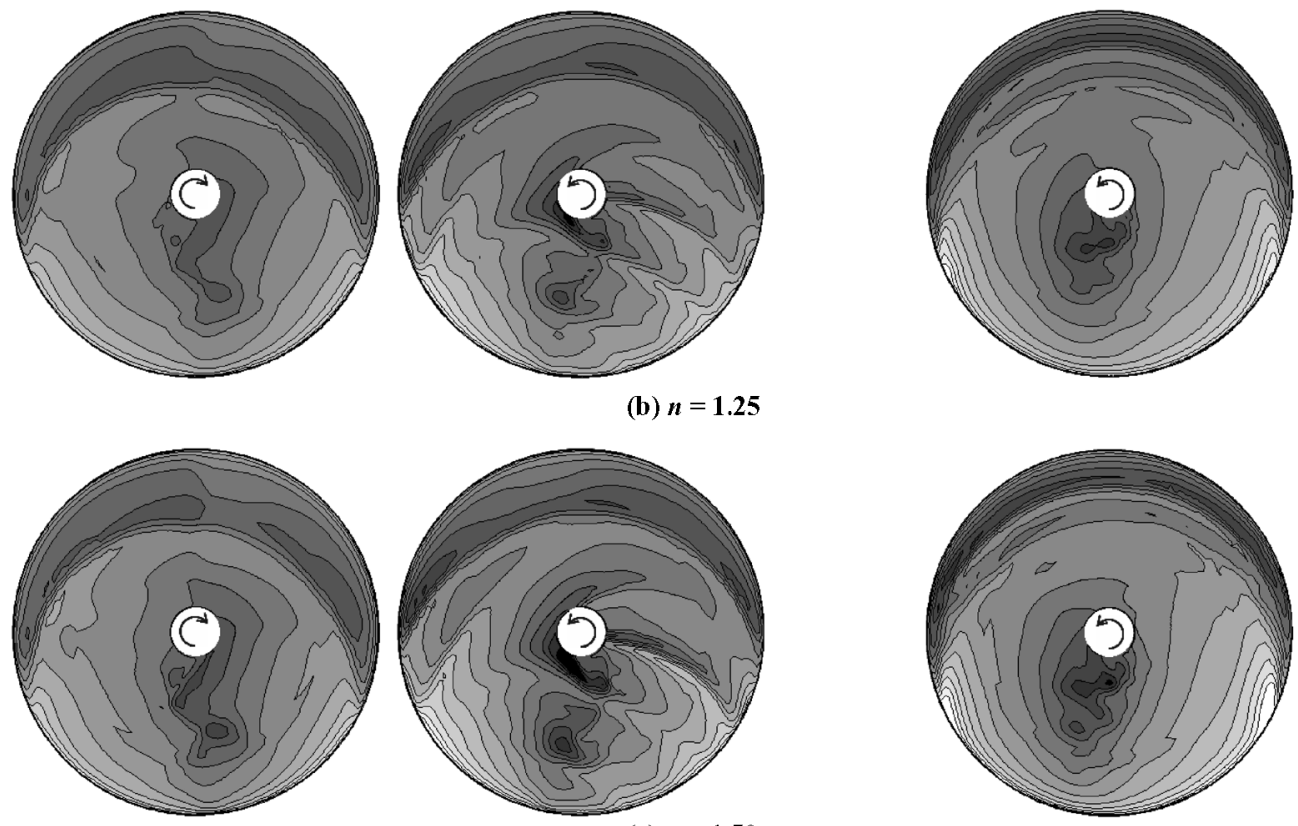

(c) $n=1.50$

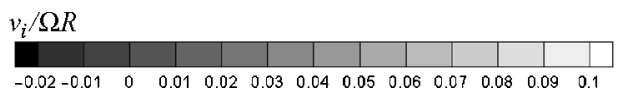

Fig. 23. Spanwise inflow variation experienced by a single blade during one revolution at various load factors (far left: upper rotor of coaxial system; left: lower rotor of coaxial system; right: equivalent conventional rotor).

the reduced inflow just upwind of the primary BVI on the forward part of the rotor disk, but this advantage is negated by the greater lateral symmetry of the composite load distribution on the rear of the coaxial system. This symmetry results in lower induced power than is given by the interaction between the highly loaded retreating blade and the developing supervortex on the left edge of the conventional rotor. Inboard, the power advantage is shared almost equally between the coaxial system and the equivalent conventional rotor, the conventional rotor generally having a lower power requirement than the coaxial system on its advancing side, and vice versa on the retreating side. In turning flight, the situation is more strongly biased in favor of the coaxial rotor. The conventional rotor still shows the strong positive effect of the upwash just forward of the strong BVI on the front of the disk, but the positive effects of the series of interrotor BVIs on the right-hand side of the lower rotor, the influence of the root vortex on the loading on the rear of both rotors of the coaxial system, and the growing drag penalty on the conventional rotor of the increased downwash to the rear of the intrarotor BVI and downwind of the interaction with the supervortex on the retreating side, all conspire to reduce the overall induced power consumption of the coaxial system relative to the equivalent rotor.

\section{Conclusions}

The performance of a coaxial rotor in hover, in steady forward flight, and in a level, coordinated turn has been contrasted with that of an equivalent, conventional rotor with the same overall solidity, number of blades, and blade aerodynamic properties. Simulations using the VTM have allowed differences in the performance of the two systems (without undue complication from fuselage and tail rotor effects) to be investigated in terms of the profile, induced, and parasite contributions to the overall power consumed by the rotors, and to be traced to differences in the structure of the wakes of the two systems.

In hover, the coaxial system consumes less induced power than the equivalent conventional system. The wake of the coaxial system in hover is dominated, close to the rotors, by the behavior of the individual tip vortices from the two rotors as they convect along the surface of two roughly concentric, but distinct, wake tubes. The axial convection rate of the tip vortices, particularly those from the upper rotor, is significantly greater than for the tip vortices of the same rotor operating in isolation. The resultant weakening of the blade-wake interaction yields a significantly reduced induced power consumption on the outer parts of the upper rotor 


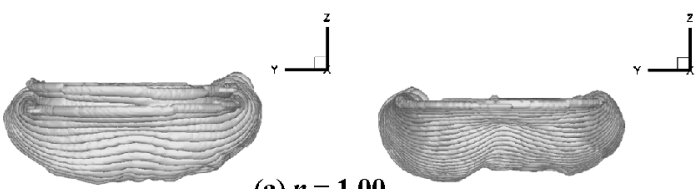

(a) $n=1.00$
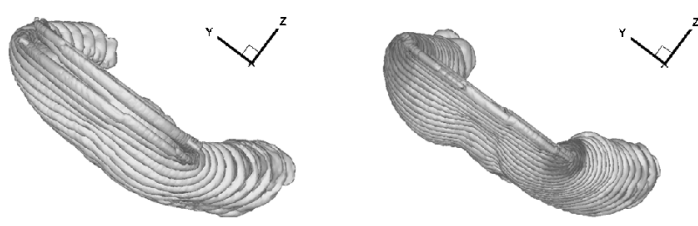

(b) $n=1.25$
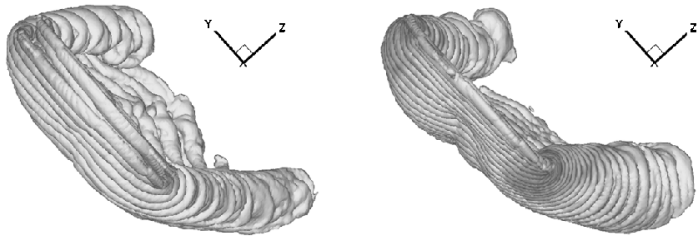

(c) $n=1.50$

Fig. 24. Wake geometry in a coordinated, level turn (front view). Left: coaxial rotor; right: equivalent rotor.

that translates into the observed benefit in terms of the induced power required by the coaxial system.

In steady, forward flight, the coaxial rotor again shows a distinct induced power advantage over its equivalent, conventional system at transitional and low advance ratios, but at high advance ratio there is very little difference between the performance of the two rotors. At a thrust coefficient of 0.0048 , the onset of stall on the retreating side of the rotors was shown to occur beyond an advance ratio of 0.28 . The rather limited maximum performance of the two systems was most likely related to the low solidity of the rotors that were simulated. With the coaxial system, the near-simultaneous stall on the retreating sides of both upper and lower rotors leads to backward flapping of both disks, although blade strike occurs at the back of the system because the upper rotor stalls more severely than the lower.

The structure of the wake generated by the coaxial and conventional systems is superficially similar at all advance ratios and shows a transition from a tube-like geometry at low advance ratio to a flattened airplanelike form at high forward speed. The formation of the wake of the coaxial rotor at posttransitional advance ratio involves an intricate process whereby the vortices from both upper and lower rotors wind around each other to create a single, merged pair of supervortices downstream of the rotor. The loading on the lower rotor is strongly influenced by interaction with the wake from the upper rotor, and there is also evidence on both rotors of intrarotor wake interaction especially at low advance ratio. In comparison, the inflow distribution on the conventional rotor, since the interrotor blade-vortex interactions are absent, is simpler in structure.

Simulations of a wind-up turn at constant advance ratio again show the coaxial rotor to possess a distinct advantage over the conventional system - a reduction in power of about $8 \%$ for load factors between 1.0 and 1.7 is observed at an advance ratio of 0.12 and a level flight thrust coefficient of 0.0048 . As in forward flight, the improved performance of the coaxial rotor results completely from a reduction in the induced power required by the system relative to the conventional rotor. This advantage is offset to a certain degree by the enhanced vibration of the coaxial system during the turn compared to the conventional system.

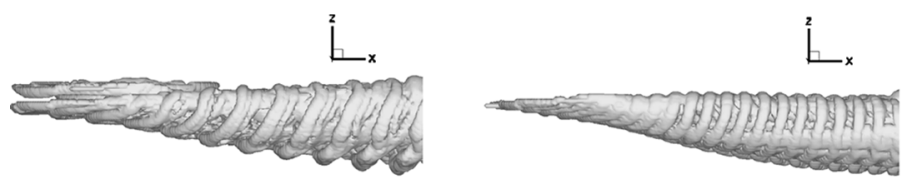

(a) $n=1.00$

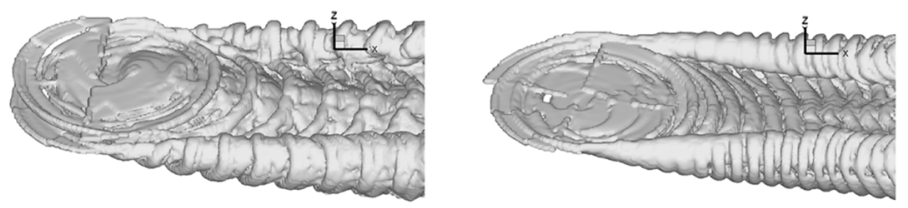

(b) $n=1.25$

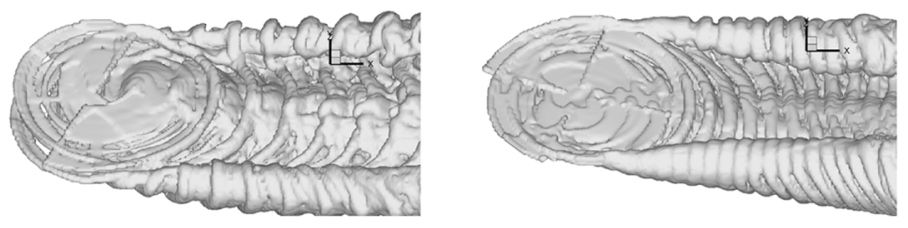

(c) $n=1.50$

Fig. 25. Wake geometry in a coordinated, level turn (side view). Left: coaxial rotor; right: equivalent rotor.

As in steady level flight, the turn performance is limited by stall and, in the coaxial system, by subsequent blade strike, at a load factor of about 1.7 for the low-solidity rotors that were used in this study. The inflow distribution on the rotors is subtly different to that in steady, level flight, and a progressive rearward shift in the positions of the interactions between the blades and their vortices with increasing load factor appears to be induced principally by the effects of the curvature of the trajectory on the geometry of the wake. Results for the simulated wind-up turn are demonstrated to be very similar to those for steady turns at the same load factor, and right-hand turns (given that the conventional rotor, and the lower rotor of the coaxial system, rotate in counterclockwise sense when viewed from above) appear to require marginally (1\%-2\%) more power than left-hand turns (the asymmetry is less marked for the coaxial system than for the equivalent, conventional rotor).

The observed differences in induced power required by the coaxial system and the equivalent, conventional rotor originate in subtle differences in the loading distribution on the two systems that are primarily associated with the pattern of blade-vortex interactions on the rotors. The beneficial properties of the coaxial rotor in forward flight and in steady turns appear to be a consequence of the somewhat greater lateral symmetry of its loading compared to the conventional system. This symmetry allows the coaxial configuration to avoid, to a small extent, the drag penalty associated with the high loading on the retreating side of the conventional rotor. It is important, though, to acknowledge the subtlety of the effects that lead to the reduced induced power requirement of the coaxial system. The computations presented here suggest that the benefits of the coaxial system do not come about merely through a broad redistribution in the loading on the system, as might be captured by a very simple model for rotor performance in which the presence of localized blade-vortex interactions is wholly neglected (e.g., blade element-momentum theory), but rather through the effect this shift in loading has in modifying the character and strength of the localized interaction between the developing supervortices and the highly loaded blade-tip regions of the rotors. Reliable prediction of these effects is well beyond the scope of simple models and is absolutely dependent on accurate prediction of the detailed structure of the rotor wake. 

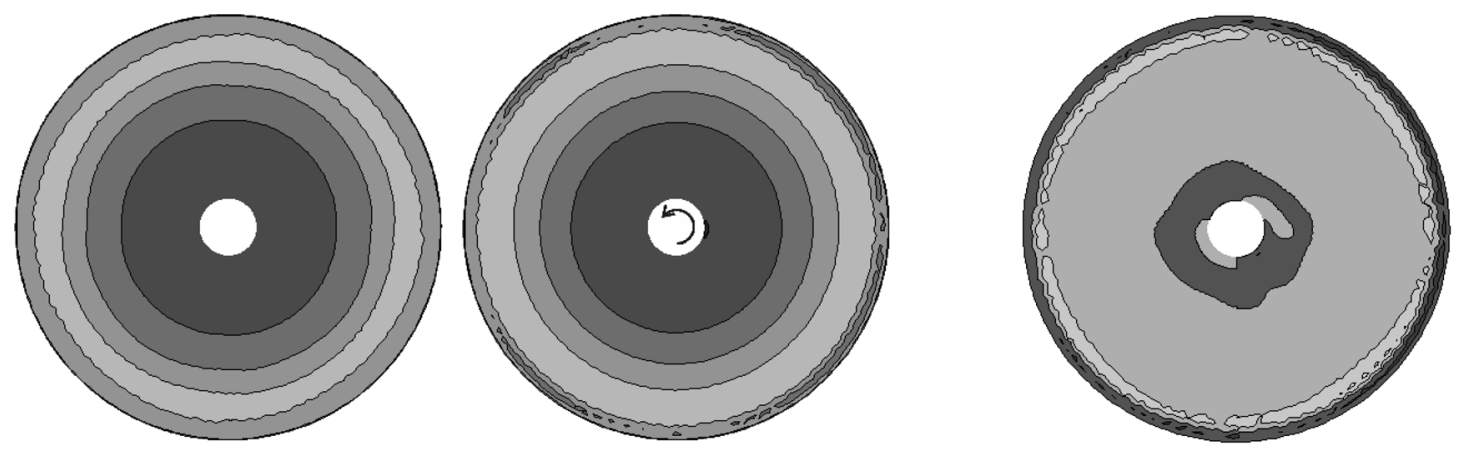

(a) Hover $\left(C_{T}=0.0048\right)$
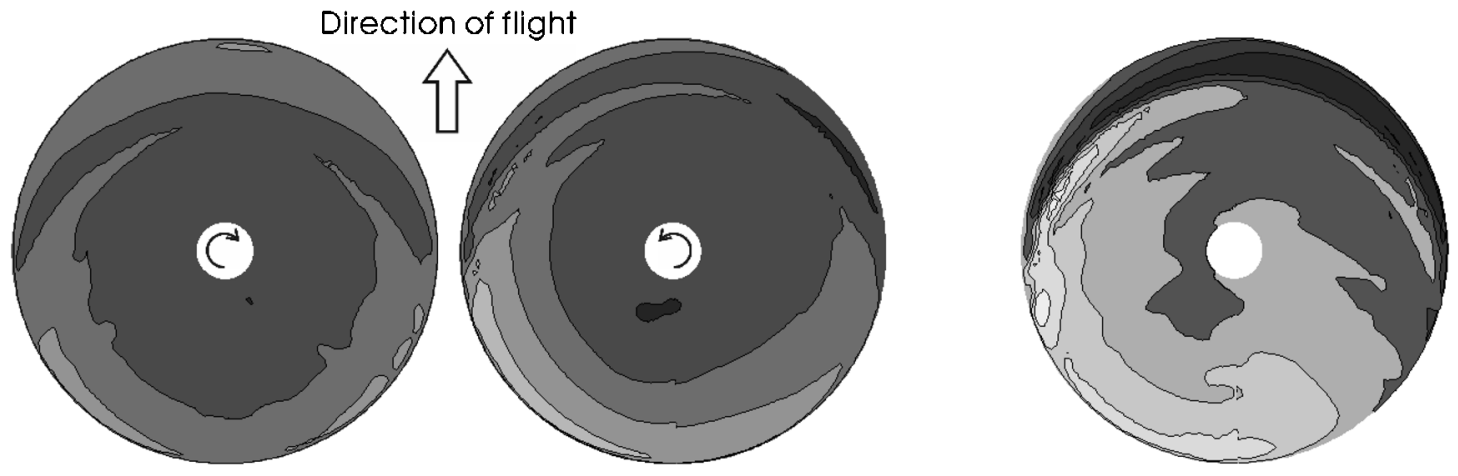

(b) Forward flight $(\mu=0.12, n=1.0)$
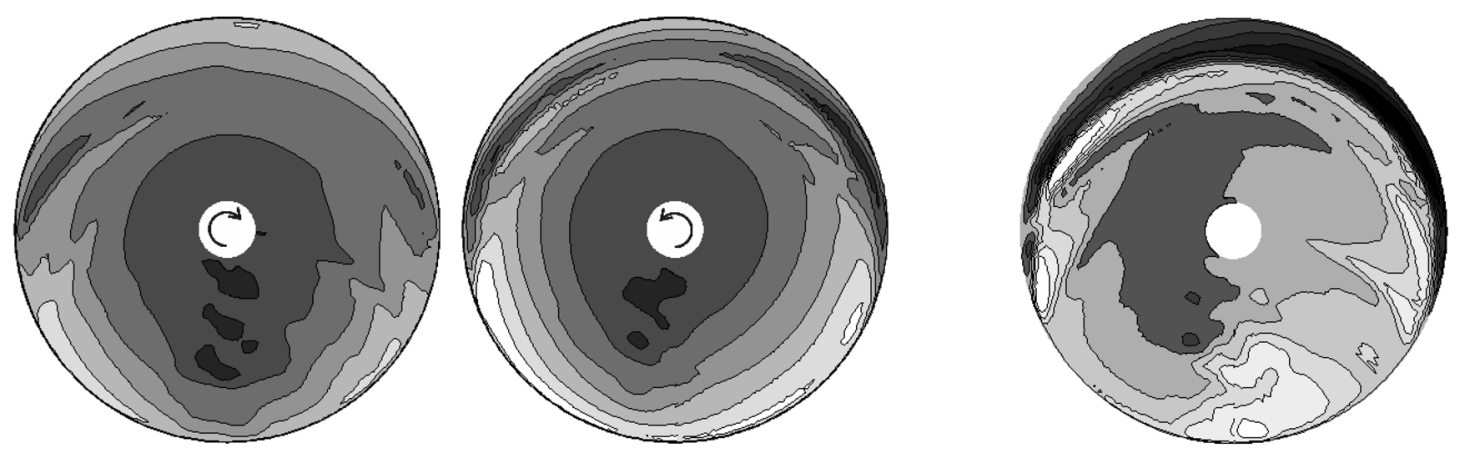

(c) Level turn $(\mu=0.12, n=1.5)$
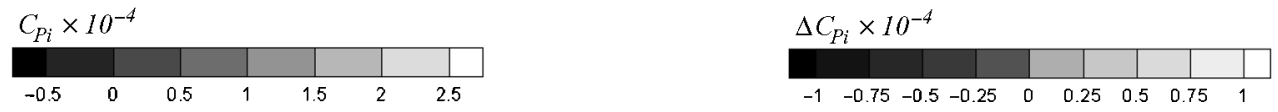

Fig. 26. Spanwise induced power variation experienced by a single blade during one revolution at various load factors (far left: coaxial system averaged over the two rotors; left: equivalent conventional rotor system; right: difference between equivalent conventional rotor system and averaged coaxial system).

\section{Acknowledgments}

This work was partially funded by the Royal Aeronautical Society via a Centennial Scholarship Award to one of the authors (HWK). Both authors would like to acknowledge their fruitful interactions with John Perry during the planning of the research that led to this paper.

\section{References}

${ }^{1}$ Coleman, C. P., "A Survey of Theoretical and Experimental Coaxial Rotor Aerodynamic Research,” NASA TP-3675, March 1997.
${ }^{2}$ Brown, R. E., "Rotor Wake Modeling for Flight Dynamic Simulation of Helicopters," AIAA Journal, Vol. 38, (1), January 2000, pp. 5763.

${ }^{3}$ Brown, R. E., and Line, A. J., "Efficient High-Resolution Wake Modeling Using the Vorticity Transport Equation," AIAA Journal, Vol. 43, (7), April 2005, pp. 1434-1443.

${ }^{4}$ Kim, H. W., and Brown, R. E., "A Rational Approach to Comparing the Performance of Coaxial and Conventional Rotors," Journal of the American Helicopter Society, 55, 012003 (2010).

${ }^{5}$ Harrington, R. D., "Full-Scale-Tunnel Investigation of the StaticThrust Performance of a Coaxial Helicopter Rotor," NACA TN-2318, March 1951. 
${ }^{6}$ Leishman, J. G., Principles of Helicopter Aerodynamics, 2nd edition, Cambridge University Press, Cambridge, UK, 2006.

${ }^{7}$ Landgrebe, A. J., "The Wake Geometry of a Hovering Helicopter Rotor and Its Influence on Rotor Performance," Journal of the American Helicopter Society, Vol. 17, (4), October 1972, pp. 215.

${ }^{8}$ Leishman, J. G., and Ananthan, S., "Aerodynamic Optimization of a Coaxial Proprotor," American Helicopter Society 62nd Annual Forum Proceedings, Phoenix, AZ, May 9-10, 2006.

${ }^{9}$ Paglino, V. M., "Forward Flight Performance of a Coaxial Rigid Rotor," American Helicopter Society 27th Annual Forum Proceedings, Washington, DC, May 13-15, 1971.
${ }^{10}$ Dingeldein, R. C., "Wind-Tunnel Studies of the Performance of Multirotor Configurations," NACA TN-3236, August 1954.

${ }^{11}$ Bhagwat, M. J., and Leishman, J. G., "On the Aerodynamic Stability of Helicopter Rotor Wakes," American Helicopter Society 56th Annual Forum Proceedings, Virginia Beach, VA, May 2-4, 2000.

${ }^{12}$ Newman, S., Brown, R., Perry, J., Lewis, S., Orchard, M., and Modha, A., "Predicting the Onset of Wake Breakdown for Rotors in Descending Flight," Journal of the American Helicopter Society, Vol. 48, (1), January 2003, pp. 28-38.

${ }^{13}$ Anikin, V. A., "Aerodynamic Feature of a Coaxial Rotor Helicopter," Paper no. 66, 17th European Rotorcraft Forum, Berlin, Germany, September 24-27, 1991. 